\title{
A phase-field/gradient damage model for brittle fracture in elastic-plastic solids
}

\author{
Fernando P. Duda ${ }^{\mathrm{a}, *}$, A. Ciarbonetti ${ }^{\mathrm{b}, \mathrm{d}}$, P.J. Sánchez ${ }^{\mathrm{b}, \mathrm{c}}$, A.E. Huespe ${ }^{\mathrm{b}}$ \\ ${ }^{a}$ Programa de Engenharia Mecânica/COPPE, Universidade Federal do Rio de Janeiro, \\ Cidade Universitária, Rio de Janeiro, Brazil. \\ ${ }^{b}$ CIMEC-UNL-CONICET, Güemes 3450, CP 3000 Santa Fe, Argentina. \\ ${ }^{c}$ GIMNI-UTN-FRSF, Lavaisse 610, CP 3000 Santa Fe, Argentina. \\ ${ }^{d}$ Autoridad Regulatoria Nuclear, Argentina.
}

\begin{abstract}
The formulation of a phase-field continuum theory for brittle fracture in elastic-plastic solids and its computational implementation are presented in this contribution. The theory is based on a virtual-power formulation in which two additional and independent kinematical descriptors are introduced, namely the phase-field and the accumulated plastic strain. Further, it incorporates irreversibility of both phase-field and plastic strain evolutions by introducing suitable constraints and by carefully heeding the influence of those constraints on the kinetics underlying microstructural changes associated with plasticity and fracture.

The numerical implementation employs the finite-element method for spatial discretization and a splitting scheme with sub-stepping for the time integration. To illustrate its potential utility, we apply the model to a number of well known linear, as well as non-linear, fracture mechanics problems.

The described phase-field model, coupled with plasticity, provides a feasible technique to analyzing crack initiation and the subsequent crack growth resistance only if the length scale parameter included in the phase-field model is finite and treated as a material parameter which should be properly characterized.
\end{abstract}

Keywords: fracture; elastoplasticity; phase-field; gradient damage mechanics.

\section{INTRODUCTION}

In classical fracture mechanics, fracture process can occur only at the crack tips. Under this premise, crack growth is driven by the near tip $J$-integral (c.f. Rice [1]; Gurtin and Podio-Guidugli [2]; Tillberg et al. [3]), a quantity that coincides with the energy release rate in the case of elastic materials. In situations involving elastic-plastic solids, it may happen that the calculated near tip $J$-integral vanishes (Rice [4], Simha et al. [5], Brocks et al. [6]), which implies that there is no

* Corresponding author

Email addresses: duda@mecanica.coppe.ufrj.br (Fernando P. Duda), angelodata@gmail.com (A. Ciarbonetti), psanchez@intec.unl.edu.ar (P.J. Sánchez), ahuespe@intec.unl.edu.ar (A.E. Huespe) 
driving force for crack growth. Hence, according to classical approach, fracture can not occur for such situations. The physical explanation for this paradox is that, contrary to the predicated by the classical approach, fracture events take place ahead the crack tip, in a finite region called the fracture process zone. Therefore, fracture mechanics models for crack problems in elastic-plastic solids must reflect the presence of a finite size fracture process zone.

Process zone description requires the modeling of material softening induced by the nucleation, growth and coalescence of microdefects - microcracks or microvoids depending on whether the underlying fracture mechanism is brittle or ductile-, which brings to the scene the issue of material length scales. Be that as it may, two families of fracture mechanics models stand out, cohesive zone models and continuum damage models (c.f. Brocks et al. [6]). A different strategy was given by Stumpf et al. [7], who used the notion of configurational forces to describe the evolution of a fracture process zone in ductile materials. In cohesive models, fracture events are assumed to take place in a surface and are described by cohesive laws. Within this category, we include the methodologies using strong discontinuity kinematics as an artifact to represent cracks such as it was shown in Simo et al. [8], Belytschko and Gracie [9], etc., and particularly, the continuum strong discontinuity approach by Oliver and coworkers (Oliver et al. [10]), Huespe et al. [11] , Huespe et al. [12]. On the other hand, continuum damage models consider the process zone as a region in which damage, described trough a continuum variable, accumulates. A material length scale parameter is innate to cohesive zone models, but, it is absent in conventional continuum damage formulations. This has led to the development of enhanced damage theories, including nonlocal and gradient theories for damage (c.f. Pijaudier-Cabot and Bazant [13], Frémond and Nedjar [14] and the references therein).

Continuum damage theories give rise to a definition of cracks as small zones of high gradients of rigidity and strength. In those zones, all fields remain continuous when critical conditions of damage are reached (e.g. Lemaitre [15]). This is exactly the view adopted in the phase-field approach to fracture, in which case damage is measured by a scalar field, the phase-field, as first proposed by Aranson et al. [16]. The phase-field approach to fracture, which may be regarded as a legitimate continuum gradient damage theory, has been extensively used to describe crack propagation in elastic solids (Spatschek et al. [17]). In this case, it can be viewed either as a regularization or as an extension of the classical theory of brittle fracture. In fact, it can be shown that the length parameter carried by the theory may be viewed as a regularizing parameter in the sense that when it goes to zero, either the classical approach (Silva Jr. et al. [18], Hakim and Karma [19]) or the variational approach (Francfort and Marigo [20]) to fracture in elastic solids is recovered. On the other hand, a finite value of this length parameter allows the description of effects that are not accounted for by the classical theory of brittle fracture, such as branching and oscillatory instabilities (c.f. Karma and Lobkovsky [21], Henry and Levine [22]).

In this paper, we develop the formulation and the numerical implementation of a small-strain continuum theory for brittle fracture in elastic-plastic solids. Typically, this failure mechanism can be observed in metal fracture below the transition temperature displaying transgranular cleavage. In this case, contained plastic deformation can take place close to notch roots. Failure criteria based on a critical stress have been proposed in the past to analyze this kind of fracture (Ritchie et al. [23]).

The formulation is carried out within the framework of continua with microstructure (e.g. Capriz [24]). Accordingly, fracture and plasticity are accounted for by two additional kinematical descriptors, the phase-field and, the accumulated plastic strain, along with the corresponding 
microforce systems. After invoking the principle of virtual power, we arrive at the basic balances of the theory, namely the standard force and moment balances, and two microforce balances. Following Fried and Gurtin [25], we introduce an energy imbalance representing a mechanical version of the second law of thermodynamics, which is used as a guide for developing a thermodynamically compatible constitutive relations. On combining these relations with the balances, we arrive at a system of coupled equations governing the evolution of displacement, phase-field and accumulated plastic strain. A special version of the theory is presented with a view towards the description of rate-independent brittle fracture in conventional elastic-plastic solids. Its numerical implementation is based on the finite element method for spatial discretization, where the displacement and phase-field are interpolated, and a staggered scheme, similar to that proposed by Bourdin et al. [26] and Miehe et al. [27], for time integration of the resulting semi-discrete evolution equations. Similar works linking gradient damage with plasticity (c.f. Nedjar [28]), as well assuming large deformation (c.f. Aslan et al. [29], Saanouni and Hamed [30], and references cited therein), have been previously published in the literature.

The numerical assessment of the model is presented in two main parts. In the first part, we show that the methodology provides a correct asymptotic solution to the sharp-crack elastic problem as long as the regularization parameter of the phase-field model goes to zero. Similar analysis have been previously presented in the literature. However, as a contribution of this paper, in sub-section 4.1.2 and 4.1.3 we emphasize the issues related to the numerical analysis of the dissipated energy of crack propagation processes. Particularly, we study the sensitivity of the model parameters for capturing the correct dissipated energy according with adopted material fracture energy. Two standard specimens are loaded until reaching the complete loss of the structural load capacity and, after very careful numerical analysis, we draw some conclusions about these issues.

A more significant contribution of the paper is presented in the second part of Section 4, where the problem of crack propagation in mode I under small-scale yielding condition is analyzed. The phase-field model interacting with a plastic deformation process is assessed, and solutions furnished by this model with those obtained using a cohesive model are compared. Also, the conditions to be satisfied by the length parameter of the phase field model in order to extract valid results are evaluated. The analysis shows that the advance of the pre-existent crack involves, in some cases, the following steps: 1) formation of a new crack ahead the original crack tip; 2) propagation of the new crack towards, and also away, the tip of the original crack; 3) extension of original crack when its tip is reached by the new crack. This implies the crack extension can occur in a discontinuous manner. This mechanism has been invoked for explaining brittle fracture in elastic-plastic solids (Tetelman and McEvily [31]; Kfouri [32]; Kfouri and Miller [33], Ritchie et al. [23]). Discontinuous crack growth can also occur within the context of ductile fracture. See, for instance, the studies carried out by Levitas [34] and Idesman et al. [35].

\section{Elastoplasticity and fracture}

We now formulate a phase-field/gradient damage theory for fracture in elastic-plastic solids under small-strains and isothermal conditions. The theory is based on a virtual-power formulation in which two additional and independent kinematical descriptors are introduced, namely the phasefield and the accumulated plastic strain. Attention is confined to isotropic behavior and conventional plasticity. The treatment of plasticity adopted here is akin to the streamlined version of 
the virtual-power principle based on codirectionality presented in Gurtin et al. [36] where the accumulated plastic strain is an independent kinematical descriptor. Following Silva Jr. et al. [18], the theory developed here incorporates irreversibility of both: phase-field and accumulated plastic strain evolutions. This is achieved by introducing suitable constraints and by carefully heeding the influence of those constraints on the kinetics underlying microstructural changes associated with plasticity and fracture.

\subsection{Preliminaries}

Let $\mathcal{B}$ be a body identified with the region it occupies in a fixed reference configuration. Let $\mathbf{u}$ denote the displacement field, $\nabla \mathbf{u}$ and $\nabla \mathbf{u}^{\top}$ the displacement gradient and its transpose, and $\mathbf{E}$ the infinitesimal strain tensor, which is defined by:

$$
\mathbf{E}=\frac{\nabla \mathbf{u}+\nabla \mathbf{u}^{\top}}{2}
$$

To account for the fact that the total deformation is the outcome of elastic and plastic contributions, the additive decomposition of the strain $\mathbf{E}$ into its elastic $\mathbf{E}_{\mathrm{e}}$ and plastic $\mathbf{E}_{\mathrm{p}}$ parts is introduced:

$$
\mathbf{E}=\mathbf{E}_{\mathrm{e}}+\mathbf{E}_{\mathrm{p}}
$$

The plastic strain $\mathbf{E}_{\mathrm{p}}$ is traceless, that is, $\operatorname{tr} \mathbf{E}_{\mathrm{p}}=0$, and satisfies the equation

$$
\dot{\mathbf{E}}_{\mathrm{p}}=\dot{e} \mathbf{N}
$$

with

$$
\dot{e}=\left|\dot{\mathbf{E}}_{\mathrm{p}}\right|, \quad|\mathbf{N}|=1, \quad \operatorname{tr}(\mathbf{N})=0 .
$$

Henceforth, $e$ is the accumulated plastic strain and $\mathbf{N}$ is the plastic orientation tensor.

\section{Remarks:}

- The assumptions represented by equations (2), (3), and (4) could be introduced later, in the section devoted to the constitutive theory. However, they were introduced at this stage for the sake of convenience only.

To describe fracture, we introduce the phase-field $\varphi$ which takes values on the interval $[0,1]$. If $\varphi=0$ at a point, then that point is unfractured. If $\varphi=1$ at some other point, then that point is fractured. Values of $\varphi$ between zero and one correspond to partially fractured material. Ideally, such values should be confined to transition zones that represent cracks.

In this paper we treat the accumulated plastic strain $e$ and the phase-field $\varphi$ as extra kinematical descriptors, whereas the plastic orientation tensor $\mathbf{N}$ is assumed to be constitutively prescribed. Thus, the kinematics of $\mathcal{B}$ is defined by the fields $\mathbf{u}, e$, and $\varphi$. The corresponding realizable velocities are denoted by $\dot{\mathbf{u}}, \dot{e}$ and $\dot{\varphi}$, whereas the virtual velocities are denoted by $\tilde{\mathbf{u}}, \tilde{e}$ and $\tilde{\varphi}$. The three force balances, one for each kinematical descriptor are deduced next. 


\subsection{Force balances}

We assume that the dynamics of $\mathcal{B}$ is specified in terms of a pair of linear, bounded, and continuous functionals, referred to as the internal and external virtual power expenditures. These are defined for any part $\mathcal{P}$, with boundary $\partial \mathcal{P}$, of $\mathcal{B}$ and a given collection $(\tilde{\mathbf{u}}, \tilde{e}, \tilde{\varphi}) \in \mathcal{V}$, where $\mathcal{V}$ is the vector space of virtual velocities and, for a given time instant, it is assumed that the list $(\dot{\mathbf{u}}, \dot{e}, \dot{\varphi}) \in \mathcal{V}$. For any part $\mathcal{P} \subset \mathcal{B}$, the internal virtual power is defined by

$$
\mathcal{W}^{\operatorname{int}}(\mathcal{P} ; \tilde{\mathbf{u}}, \tilde{e}, \tilde{\varphi}):=\int_{\mathcal{P}}(\mathbf{S} \cdot \nabla \tilde{\mathbf{u}}+\boldsymbol{\xi} \cdot \nabla \tilde{\varphi}+\gamma \tilde{e}+\pi \tilde{\varphi}) \mathrm{d} v
$$

where the macrostress tensor $\mathbf{S}$ and the microstress $\boldsymbol{\xi}$ represent interactions of first order, whereas the internal microforces densities $\gamma$ and $\pi$ represent interactions of zeroth order. Note that since we are interested in conventional plasticity only, which by its turn is a local theory, we do not consider first order interaction for $\nabla \tilde{e}$. Otherwise, accounting for this interaction would provide a foundation upon which to build gradient plasticity theories. Further, for any part $\mathcal{P} \subset \mathcal{B}$, the external virtual power is defined by

$$
\mathcal{W}^{\operatorname{ext}}(\mathcal{P} ; \tilde{\mathbf{u}}, \tilde{e}, \tilde{\varphi}):=\int_{\partial \mathcal{P}}\left(\mathbf{s} \cdot \tilde{\mathbf{u}}+\xi_{\partial \mathcal{P}} \tilde{\varphi}\right) \mathrm{d} a+\int_{\mathcal{P}}\left(\mathbf{b} \cdot \tilde{\mathbf{u}}+\gamma^{\operatorname{ext}} \tilde{e}+\pi^{\operatorname{ext}} \tilde{\varphi}\right) \mathrm{d} v
$$

where $\mathbf{s}$ and $\xi_{\partial \mathcal{P}}$ represent contact interactions and $\mathbf{b}, \gamma^{\text {ext }}$ and $\pi^{\text {ext }}$ interactions at a distance. The standard body force $\mathrm{b}$ is presumed to include inertia, that is,

$$
\mathbf{b}=\mathbf{b}_{0}-\rho \ddot{\mathbf{u}}
$$

in an inertial frame. Here, $\mathbf{b}_{\mathbf{0}}$ is the non-inertial contribution to $\mathbf{b}$ and $\rho$ is the mass density.

The principle of virtual power, which is assumed to hold at each fixed time, states that, for any given part $\mathcal{P} \subset \mathcal{B}$ :

1. The internal and external virtual powers are balanced, that is,

$$
\mathcal{W}^{\text {int }}(\mathcal{P} ; \tilde{\mathbf{u}}, \tilde{e}, \tilde{\varphi})=\mathcal{W}^{\operatorname{ext}}(\mathcal{P} ; \tilde{\mathbf{u}}, \tilde{e}, \tilde{\varphi})
$$

for each admissible virtual velocity $(\tilde{\mathbf{u}}, \tilde{e}, \tilde{\varphi})$.

2. The internal virtual power is frame-indifferent, that is,

$$
\mathcal{W}^{\text {int }}(\mathcal{P} ; \tilde{\mathbf{u}}, \tilde{e}, \tilde{\varphi})=\mathcal{W}^{\text {int }}\left(\mathcal{P} ; \tilde{\mathbf{u}}^{*}, \tilde{e}^{*}, \tilde{\varphi}^{*}\right)
$$

where

$$
\mathbf{u}^{*}=\mathbf{u}+\mathbf{a}+\mathbf{W r}, \quad \tilde{e}^{*}=\tilde{e}, \quad \tilde{\varphi}^{*}=\tilde{\varphi} .
$$

Here, a and $\mathbf{W}$ are arbitrary spatially constant vector and skew tensor fields, respectively, and $\mathbf{r}=\mathbf{x}-\mathbf{o}$ is the position vector at a point $\mathbf{x}$ in $\mathcal{P}$, with $\mathbf{o}$ being the point at the origin.

This principle implies the force balances:

- Standard force balance

$$
\operatorname{div} \mathbf{S}+\mathbf{b}=\mathbf{0} \quad \text { on } \mathcal{P} \quad \text { and } \quad \mathbf{S n}=\mathbf{s} \quad \text { on } \partial \mathcal{P}
$$

where $\mathbf{S}$ is symmetric thanks to the frame-indifference of the internal power; 
- Phase-field microforce balance

$$
\operatorname{div} \boldsymbol{\xi}-\pi+\pi^{\text {ext }}=0 \quad \text { on } \mathcal{P} \quad \text { and } \quad \boldsymbol{\xi} \cdot \mathbf{n}=\xi_{\partial \mathcal{P}} \quad \text { on } \partial \mathcal{P}
$$

- Plastic-strain microforce balance

$$
-\gamma+\gamma^{\mathrm{ext}}=0 \quad \text { on } \mathcal{P}
$$

where $\mathbf{n}$ is the exterior unit vector normal to $\partial \mathcal{P}$. Notice that $(11)_{1}$ represents the local form of the standard force balance, whereas $(11)_{2}$ the standard traction condition, with $\mathbf{S}$ and $\mathbf{b}$ representing the standard stress and body force, respectively. Likewise, $(12)_{1}$ gives the local form of the microforce balance equation associated to the phase-field and $(12)_{2}$ the corresponding traction condition, with $\boldsymbol{\xi}, \pi$, and $\pi^{\text {ext }}$ representing the microstress, internal microforce, and external microforce, which are associated to processes leading to fracture as described by the phase-field $\varphi$. Since a zeroth-order description has been adopted for plasticity, there is no traction condition in (13).

\subsection{Energy imbalance}

In addition to the force balances introduced before, we impose the first and second laws of thermodynamics via an energy imbalance. This imbalance requires that the rate of change of the free energy of any $\mathcal{P}$ does not exceed the inflow of energy to $\mathcal{P}$ due to the power expended on $\mathcal{P}$ by external agencies. Specifically, introducing the free-energy density $\psi$ per unit volume, the law of energy imbalance for $\mathcal{P}$ reads

$$
\frac{\mathrm{d}}{\mathrm{d} t} \int_{\mathcal{P}} \psi \mathrm{d} v \leq \mathcal{W}^{\text {ext }}(\mathcal{P}, \dot{\mathbf{u}}, \dot{e}, \dot{\varphi})
$$

Bearing in mind that $\mathcal{P}$ is arbitrary, making use of the power balance (8), and recalling that $\mathbf{S}$ is symmetric, we find the integral statement (14) of energy imbalance has the equivalent point-wise version

$$
\dot{\psi}-\mathbf{S} \cdot \dot{\mathbf{E}}-\boldsymbol{\xi} \cdot \nabla \dot{\varphi}-\gamma \dot{e}-\pi \dot{\varphi} \leq 0 .
$$

In view of (2), (3), and (4) $)_{3}$, this implies that

$$
\dot{\psi}-\mathbf{S} \cdot \dot{\mathbf{E}}_{\mathrm{e}}-\boldsymbol{\xi} \cdot \nabla \dot{\varphi}-\bar{\gamma} \dot{e}-\pi \dot{\varphi} \leq 0,
$$

where

$$
\bar{\gamma}=\gamma+\tau, \quad \tau=\mathbf{S}_{0} \cdot \mathbf{N},
$$

where $\tau$ is the resolved stress obtained by projecting $\mathbf{S}_{0}$, the deviatoric part of $\mathbf{S}$, along the plastic flow direction. 


\subsection{Constitutive theory}

The structure of the inequality (16) dictates certain natural choices for dependent and independent constitutive variables. Before taking this point, it is crucial to decide if it is possible or not to choose arbitrarily $\dot{\mathbf{E}}_{\mathrm{e}}, \dot{e}, \dot{\varphi}$, and $\nabla \dot{\varphi}$. When this choice is limited, $\mathcal{B}$ is subjected to internal constraints (Capriz [24]). If this is the case, the standard practice of dealing with internal constraints dictates that $\mathbf{S}, \boldsymbol{\xi}, \pi$ and $\bar{\gamma}$ are sums of two components, one called active and the other called reactive, that is,

$$
\mathbf{S}=\mathbf{S}_{\mathrm{a}}+\mathbf{S}_{\mathrm{r}}, \quad \boldsymbol{\xi}=\boldsymbol{\xi}_{\mathrm{a}}+\boldsymbol{\xi}_{\mathrm{r}}, \quad \pi=\pi_{\mathrm{a}}+\pi_{\mathrm{r}}, \quad \bar{\gamma}=\bar{\gamma}_{\mathrm{a}}+\bar{\gamma}_{\mathrm{r}},
$$

where constitutive relations can be assigned only for the active components. Further, the reactive contributions expend no power, that is,

$$
\mathbf{S}_{\mathrm{r}} \cdot \dot{\mathbf{E}}_{\mathrm{e}}+\boldsymbol{\xi}_{\mathrm{r}} \cdot \nabla \dot{\varphi}+\pi_{\mathrm{r}} \dot{\varphi}+\bar{\gamma}_{\mathrm{r}} \dot{e}=0
$$

for all admissible $\dot{\mathbf{E}}_{\mathrm{e}}, \dot{e}, \dot{\varphi}$, and $\nabla \dot{\varphi}$.

In this work, we assume that $\dot{\mathbf{E}}_{\mathrm{e}}$ and $\nabla \dot{\varphi}$ are unconstrained, whereas $\dot{e}$ and $\dot{\varphi}$ are such that:

$$
\dot{e} \geq 0, \quad \dot{\varphi} \geq 0
$$

These constraints represent the often invoked assumption that microstructural changes leading to plasticity and fracture are irreversible. With these assumptions, it follows from (19) that

$$
\begin{gathered}
\mathbf{S}_{\mathrm{r}}=\mathbf{0}, \quad \boldsymbol{\xi}_{\mathrm{r}}=\mathbf{0}, \\
\bar{\gamma}_{\mathrm{r}}= \begin{cases}\text { undetermined, } & \text { if } \dot{e}=0, \\
0, & \text { if } \dot{e}>0,\end{cases}
\end{gathered}
$$

and that

$$
\pi_{\mathrm{r}}= \begin{cases}\text { undetermined, } & \text { if } \dot{\varphi}=0, \\ 0, & \text { if } \dot{\varphi}>0 .\end{cases}
$$

Therefore, whereas $\mathbf{S}$ and $\boldsymbol{\xi}$ are constitutively assigned, $\bar{\gamma}$ and $\pi$ are constitutively assigned only for $\dot{e}>0$ and $\dot{\varphi}>0$, respectively.

\subsubsection{Constitutive response functions}

Suppose that $\dot{e}>0$ and $\dot{\varphi}>0$. Guided by the structure of the inequality (16), we allow for possible constitutive dependencies of $\psi, \mathbf{S}, \bar{\gamma}, \pi$, and $\boldsymbol{\xi}$ on $\mathbf{E}_{\mathrm{e}}, e, \varphi, \nabla \varphi, \dot{e}$, and $\dot{\varphi}$. Granted the availability of external forces needed to ensure the satisfaction of the local balances $(11)_{1},(12)_{1}$ and (13), a straightforward application of an argument first employed by Coleman and Noll [37] shows that $\psi$ must be independent of $\dot{\varphi}$,

$$
\psi=\hat{\psi}\left(\mathbf{E}_{\mathrm{e}}, e, \varphi, \nabla \varphi\right)
$$

that $\mathbf{S}$ and $\boldsymbol{\xi}$ must be given by equations of state

$$
\left.\begin{array}{c}
\mathbf{S}=\frac{\partial \hat{\psi}\left(\mathbf{E}_{\mathrm{e}}, e, \varphi, \nabla \varphi\right)}{\partial \mathbf{E}_{\mathrm{e}}}, \\
\boldsymbol{\xi}=\frac{\partial \hat{\psi}\left(\mathbf{E}_{\mathrm{e}}, e, \varphi, \nabla \varphi\right)}{\partial \nabla \varphi},
\end{array}\right\}
$$


that $\bar{\gamma}$ and $\pi$ must be a sum of the form

$$
\bar{\gamma}=\frac{\partial \hat{\psi}\left(\mathbf{E}_{\mathrm{e}}, e, \varphi, \nabla \varphi\right)}{\partial e}+\hat{\gamma}_{\mathrm{dis}}\left(\mathbf{E}_{\mathrm{e}}, e, \varphi, \nabla \varphi, \dot{e}, \dot{\varphi}\right)
$$

and

$$
\pi=\frac{\partial \hat{\psi}\left(\mathbf{E}_{\mathrm{e}}, e, \varphi, \nabla \varphi\right)}{\partial \varphi}+\hat{\pi}_{\mathrm{dis}}\left(\mathbf{E}_{\mathrm{e}}, e, \varphi, \nabla \varphi, \dot{e}, \dot{\varphi}\right),
$$

and that $\hat{\gamma}_{\text {dis }}$ and $\hat{\pi}_{\text {dis }}$ must obey the residual dissipation inequality

$$
\delta=\hat{\gamma}_{\mathrm{dis}}\left(\mathbf{E}_{e}, e, \varphi, \nabla \varphi, \dot{e}, \dot{\varphi}\right) \dot{e}+\hat{\pi}_{\mathrm{dis}}\left(\mathbf{E}_{e}, e, \varphi, \nabla \varphi, \dot{e}, \dot{\varphi}\right) \dot{\varphi} \geq 0,
$$

where $\delta$ denotes the dissipation density.

Instead of determining the most general constitutive response functions $\hat{\gamma}_{\text {dis }}$ and $\hat{\pi}_{\text {dis }}$ of (28), we suppose that $\hat{\gamma}_{\text {dis }}$ is independent of $\dot{\varphi}$ and $\hat{\pi}_{\text {dis }}$ independent of $\dot{e}$. Thus, we restrict attention to solutions for which the dissipative mechanisms associated with plasticity and fracture are decoupled, in which case (28) implies the inequalities

$$
\hat{\gamma}_{\text {dis }}\left(\mathbf{E}_{\mathrm{e}}, e, \varphi, \nabla \varphi, \dot{e}\right) \dot{e} \geq 0
$$

and

$$
\hat{\pi}_{\text {dis }}\left(\mathbf{E}_{\mathrm{e}}, e, \varphi, \nabla \varphi, \dot{\varphi}\right) \dot{\varphi} \geq 0,
$$

hold separately. Henceforth, we assume that $\hat{\gamma}_{\text {dis }}$ and $\hat{\pi}_{\text {dis }}$ are given by

$$
\hat{\gamma}_{\text {dis }}\left(\mathbf{E}_{\mathrm{e}}, e, \varphi, \nabla \varphi, \dot{e}\right)=\hat{a}\left(\mathbf{E}_{\mathrm{e}}, e, \varphi, \nabla \varphi\right)+\hat{b}\left(\mathbf{E}_{\mathrm{e}}, e, \varphi, \nabla \varphi\right) \dot{e}
$$

and

$$
\hat{\pi}_{\mathrm{dis}}\left(\mathbf{E}_{\mathrm{e}}, e, \varphi, \nabla \varphi, \dot{\varphi}\right)=\hat{\alpha}\left(\mathbf{E}_{\mathrm{e}}, e, \varphi, \nabla \varphi\right)+\hat{\beta}\left(\mathbf{E}_{\mathrm{e}}, e, \varphi, \nabla \varphi\right) \dot{\varphi},
$$

where, to ensure satisfaction of (30) and (29), the response functions $\hat{a}, \hat{b}, \hat{\alpha}$ and $\hat{\beta}$ are chosen to be non-negative valued functions. The condition $\hat{b}=0$ in (31) corresponds to rate-independent plastic response, whereas the condition $\hat{\beta}=0$ in (32) corresponds to rate-independent damage response.

Now, we prescribe the constitutive equations for $\bar{\gamma}_{\mathrm{a}}$, the active part of $\bar{\gamma}$, when $\dot{e}=0$, and $\pi_{\mathrm{a}}$, the active part of $\pi$, when $\dot{\varphi}=0$. In both cases, we assume that the constitutive equations for $\psi, \mathbf{S}$ and $\boldsymbol{\xi}$ are given by (24) and (25). Since $\dot{e}=0$, thermodynamic compatibility places no restriction on constitutive responses for $\bar{\gamma}_{\mathrm{a}}$. In this sense, constitutive equations for $\bar{\gamma}_{\mathrm{a}}$ may be freely prescribed. Hereafter, we stipulate that, if $\dot{e}=0$,

$$
\bar{\gamma}_{\mathrm{a}}=\frac{\partial \hat{\psi}\left(\mathbf{E}_{\mathrm{e}}, e, \varphi, \nabla \varphi\right)}{\partial e}+\hat{a}\left(\mathbf{E}_{\mathrm{e}}, e, \varphi, \nabla \varphi\right) .
$$

By a similar reasoning, constitutive equations for $\pi_{\mathrm{a}}$ may be freely prescribed. Hereafter, we stipulate that, if $\dot{\varphi}=0$,

$$
\pi_{\mathrm{a}}=\frac{\partial \hat{\psi}\left(\mathbf{E}_{\mathrm{e}}, e, \varphi, \nabla \varphi\right)}{\partial \varphi}+\hat{\alpha}\left(\mathbf{E}_{\mathrm{e}}, e, \varphi, \nabla \varphi\right) .
$$

As will become evident, the choices (33)-(34) for $\bar{\gamma}_{\mathrm{a}}$ and $\pi_{a}$ are very convenient. In particular, it can be shown that these choices imply that $\bar{\gamma}_{\mathrm{r}}$ and $\pi_{r}$ change continuously in a process during which $\dot{e}$ and $\dot{\varphi}$ change continuously from $\dot{e}=0$ and $\dot{\varphi}=0$ to $\dot{e}>0$ and $\dot{\varphi}>0$. 


\subsubsection{Yield criterion and the plastic orientation tensor}

We now specify the yield criterion, that is, the condition under which plasticity occurs, and the plastic orientation tensor $\mathbf{N}$. The yield criterion adopted here is motivated by the microforce balance (13).

On assuming that $\gamma^{\text {ext }}=0$, the use of the relations (17), (26), (31), and (33) in the microforce balance (13) yields that

$$
\tau-y= \begin{cases}\eta \dot{e}, & \dot{e}>0, \\ \bar{\gamma}_{\mathrm{r}}, & \dot{e}=0,\end{cases}
$$

where, for sake of notation, we have introduced the quantities

$$
\eta:=\hat{b}\left(\mathbf{E}_{\mathrm{e}}, e, \varphi, \nabla \varphi\right), \quad y:=\frac{\partial \hat{\psi}\left(\mathbf{E}_{\mathrm{e}}, e, \varphi, \nabla \varphi\right)}{\partial e}+\hat{a}\left(\mathbf{E}_{\mathrm{e}}, e, \varphi, \nabla \varphi\right) .
$$

In the sequel, it is important to keep in mind the distinction between the two alternatives in (35): the first alternative is a restriction on the manner that microstructural changes leading to plasticity occur; the second alternative is an identity that determines $\bar{\gamma}_{\mathrm{r}}$ when such changes are arrested -in which case, the microforce balance is automatically satisfied.

We now turn to the specification of the yield criterion. First, we consider that $\eta>0$, that is, a rate-dependent plasticity response. In this case, $(35)_{1}$ implies that $\dot{e}>0$ only if

$$
\tau>y
$$

Here, we stipulate that condition (37) is also sufficient for $\dot{e}>0$, which means that the yield condition is given by (37). Notice that, equivalently, $\dot{e}=0$ if and only if

$$
\tau \leq y .
$$

For a rate-independent plastic response, (35) indicates that $\dot{e}>0$ only if $\tau=y$. In this case, we stipulate that (38) always must be obeyed, but now with the provision that the strict inequality is sufficient but not necessary for $\dot{e}=0$. Under these conditions, it can be shown that $\dot{e}>0$ if and only if

$$
\tau=y \quad \text { and } \quad \dot{\tau}=\dot{y} .
$$

It is easy to see that in both rate-dependent and rate-independent case, the reactive microforce $\bar{\gamma}_{\mathrm{r}}$ obeys the inequality

$$
\bar{\gamma}_{\mathrm{r}} \leq 0
$$

Notice that the rate-independent case can be obtained from the rate-dependent one in the limit as $\eta$ goes to zero (c.f. Simo and Hughes [38]).

To prescribe the plastic orientation tensor $\mathbf{N}$, we begin by observing that after taking $(17)_{2}$, (29), (31), (36), (35) into account, it follows that the plastic dissipation density is given by

$$
\left(\mathbf{S}_{0} \cdot \mathbf{N}-\frac{\partial \hat{\psi}\left(\mathbf{E}_{\mathrm{e}}, e, \varphi, \nabla \varphi\right)}{\partial e}\right) \dot{e} \geq 0
$$


Notice that $(25)_{1}$ implies that $\mathbf{S}_{0}$, the deviatoric part of $\mathbf{S}$, is a function of the list $\left(\mathbf{E}_{\mathrm{e}}, e, \varphi, \nabla \varphi\right)$. Therefore, for a given $\left(\mathbf{E}_{\mathrm{e}}, e, \varphi, \nabla \varphi\right)$, the plastic dissipation is a function of $\mathbf{N}$, whose maximum is attained at

$$
\mathbf{N}=\frac{\mathbf{S}_{0}}{\left|\mathbf{S}_{0}\right|}
$$

and hence

$$
\tau=\left|\mathbf{S}_{0}\right|
$$

Thus, if the principle of maximum dissipation is invoked, the equations above must hold. See Gurtin et al. [36] for a detailed discussion of this issue. See also Aifantis [39].

\subsubsection{Damage criterion}

Now, we turn to the issue of the damage criterion. The damage criterion adopted here is motivated by the microforce balance (12). The development follows along the same lines adopted for plasticity.

After assuming that $\pi^{\text {ext }}=0$ and using the relations $(12)_{1},(25)_{2},(27)$ and (32), it follows from the microforce balance (12) that

$$
\zeta-\alpha= \begin{cases}\beta \dot{\varphi}, & \dot{\varphi}>0 \\ \pi_{\mathrm{r}}, & \dot{\varphi}=0\end{cases}
$$

where, to simplify the notation,

$$
\beta:=\hat{\beta}\left(\mathbf{E}_{e}, e, \varphi, \nabla \varphi\right), \quad \alpha:=\hat{\alpha}\left(\mathbf{E}_{e}, e, \varphi, \nabla \varphi\right)
$$

and

$$
\zeta:=\operatorname{div}\left(\frac{\partial \hat{\psi}\left(\mathbf{E}_{e}, e, \varphi, \nabla \varphi\right)}{\partial \nabla \varphi}\right)-\frac{\partial \hat{\psi}\left(\mathbf{E}_{e}, e, \varphi, \nabla \varphi\right)}{\partial \varphi}
$$

Notice that $-\zeta$ is the variational derivative of $\hat{\psi}$ with respect to $\varphi$. Again, it is important to keep in mind the distinction between the two alternatives in (44): the first alternative is a restriction on the manner microstructural changes leading to damage occur; the second alternative is an identity that determines $\pi_{\mathrm{r}}$ when such changes are arrested -in which case, the microforce balance is automatically satisfied.

When $\beta>0$, that is, for a rate-dependent damage response, $(44)_{1}$ implies that

$$
\zeta>\alpha
$$

is a necessary condition for $\dot{\varphi}>0$. Here, we stipulate that condition is also sufficient for $\dot{\varphi}>0$, which means that damage occurs if and only (47) holds. Equivalently, no damage occurs whenever

$$
\zeta \leq \alpha
$$

is satisfied. For a rate-independent damage response, i.e., for $\beta=0,(44)_{1}$ indicates that $\zeta=\alpha$ is a necessary condition for $\dot{\varphi}>0$. In this case, we stipulate that (48) always holds, but now with the 
provision that the strict inequality is sufficient but not necessary for $\dot{\varphi}=0$. Under these conditions, it can be shown that $\dot{\varphi}>0$ if and only if

$$
\zeta=\alpha \quad \text { and } \quad \dot{\zeta}=\dot{\alpha} .
$$

It is easy to see that in both rate-dependent and rate-independent case, the reactive microforce $\pi_{\mathrm{r}}$ obeys the inequality

$$
\pi_{\mathrm{r}} \leq 0
$$

\subsection{Governing equations}

Now, we collect the results obtained so far to write the set of governing equations for the basic fields of the theory, namely $\mathbf{u}, e, \varphi, \bar{\gamma}_{\mathrm{r}}$ and $\pi_{\mathrm{r}}$. This set of equations is obtained by combining the force balances and constitutive theory, including the yield and damage criteria.

We begin by considering the governing equations for $\dot{e}$ and $\bar{\gamma}_{\mathrm{r}}$. For the sake of convenience, let us introduce the function $f$ defined by

$$
f:=\left|\mathbf{S}_{0}\right|-y
$$

with $y$ given by $(36)_{2}$. In the rate-independent case, it is easy to see that the governing equations for $\dot{e}$ and $\bar{\gamma}_{\mathrm{r}}$ are given by

$$
\dot{e} \geq 0, \quad f \leq 0, \quad \dot{e} f=0, \quad \bar{\gamma}_{\mathrm{r}}=f,
$$

whereas in the rate-dependent case they are given by

$$
\eta \dot{e}=\langle f\rangle, \quad \bar{\gamma}_{\mathrm{r}}=-\langle-f\rangle,
$$

with $\eta$ given by (36) $)_{1}$. Henceforth $\langle h\rangle$ denotes the Macaulay bracket of a scalar-valued quantity $h$, that is,

$$
\langle h\rangle= \begin{cases}0, & h \leq 0 \\ h, & h>0\end{cases}
$$

After introducing the function

$$
F=\zeta-\alpha
$$

with $\zeta$ and $\alpha$ given by (46) and (45) 2 , it can be shown that the governing equations for $\dot{\varphi}$ and $\pi_{\mathrm{r}}$ reduce to

$$
\dot{\varphi} \geq 0, \quad F \leq 0, \quad \dot{\varphi} F=0, \quad \pi_{\mathrm{r}}=F
$$

in the rate-independent case, and to:

$$
\beta \dot{\varphi}=\langle F\rangle, \quad \pi_{\mathrm{r}}=-\langle-F\rangle
$$

otherwise, where $\beta$ is defined in $(45)_{1}$.

The remaining governing equations are given by the (1), (2), (3) with $\mathbf{N}=\mathbf{S}_{0} /\left|\mathbf{S}_{0}\right|$, and the standard force balance (11) with $\mathbf{S}$ given by $(25)_{1}$. 


\subsection{Specialization}

We now specialize the constitutive response functions introduced before with a view towards the development of a simple rate-independent continuum model for fracture in elastic-plastic materials. As described below, we assume that the free energy is given as the sum of elastic, fracture and plastic contributions. Further, we consider that damage results from microcracking and manifests itself only through a reduction in the elastic energy storage capacity of the material.

Our choice for the free energy response $\hat{\psi}$ is

$$
\hat{\psi}\left(\mathbf{E}_{e}, e, \varphi, \nabla \varphi\right)=\hat{\psi}_{e}\left(\mathbf{E}_{e}, \varphi\right)+\hat{\psi}_{p}(e)+\hat{\psi}_{f}(\varphi, \nabla \varphi),
$$

with

$$
\begin{aligned}
& \hat{\psi}_{e}\left(\mathbf{E}_{e}, \varphi\right)=\left((1-\varphi)^{2}+k_{c}\right) \hat{\psi}_{e}^{+}\left(\mathbf{E}_{e}\right)+\hat{\psi}_{e}^{-}\left(\mathbf{E}_{e}\right) ; \\
& \hat{\psi}_{e}^{+}\left(\mathbf{E}_{e}\right)=\frac{1}{2}\left(\lambda\left\langle\operatorname{tr} \mathbf{E}_{e}\right\rangle^{2}+2 \mu \mathbf{E}_{e}^{+}: \mathbf{E}_{e}^{+}\right) ; \\
& \hat{\psi}_{e}^{-}\left(\mathbf{E}_{e}\right)=\frac{1}{2}\left(\lambda\left\langle-\operatorname{tr} \mathbf{E}_{e}\right\rangle^{2}+2 \mu \mathbf{E}_{e}^{-}: \mathbf{E}_{e}^{-}\right) ; \\
& \mathbf{E}_{e}^{+}=\left\langle\left(E_{e}\right)_{i}\right\rangle \mathbf{e}_{i} \otimes \mathbf{e}_{i} ; \quad \mathbf{E}_{e}^{-}=-\left\langle-\left(E_{e}\right)_{i}\right\rangle \mathbf{e}_{i} \otimes \mathbf{e}_{i} ; \\
& \hat{\psi}_{p}(e)=\frac{\sigma_{y}^{2}}{E(n+1)}\left(1+\sqrt{\frac{2}{3}} \frac{E e}{\sigma_{y}}\right)^{n+1}-\sqrt{\frac{2}{3}} \sigma_{y} e,
\end{aligned}
$$

and

$$
\hat{\psi}_{f}(\varphi, \nabla \varphi)=g_{f}\left(\frac{\varphi^{2}}{2 l}+\frac{l}{2}|\nabla \varphi|^{2}\right) .
$$

Here, $\lambda$ and $\mu$ are the Lamé parameters, $E$ is the Young's modulus, $\left(E_{e}\right)_{i}$ and $\mathbf{e}_{i}$ are the i-th principal strain and eigenvector of $\mathbf{E}_{e}$, respectively. In expressions $(59)_{2-3}$, and according with $(59)_{4}, \mathbf{E}_{e}^{+}$and $\mathbf{E}_{e}^{-}$are the spectral decomposition of the positive and negative elastic strain. The constant parameter $k_{c}$ is greater than zero $\left(k_{c}>0\right)$, and is introduced to prevent a possible illconditioning of the model when $\varphi=1$. But otherwise, it should be as small as possible to avoid an overestimation of the bulk energy. The strain hardening exponent of the plastic model is denoted $n$, the yield stress at $e=0$ is $\sigma_{y}$, the fracture energy is $g_{f}$, and $l$ is a parameter with dimension of length.

Notice that $\hat{\psi}_{e}$, the elastic contribution to the free energy, contains an interaction term between unilateral damage, which accounts for microcrack closure/opening effect, and elastic strain, and that this term is a decreasing function of the degree of degradation as described by the phase field. Thus, $\hat{\psi}_{e}$ is decomposed additively in two terms associated with the positive and negative elastic strains. Only the first component of $\hat{\psi}_{e}$ couples $\varphi$ with elastic strains. As can be seen in the following development, the coupling of $\varphi$ with $\hat{\psi}_{e}^{+}$means that the evolution of $\varphi$ is driven by $\hat{\psi}_{e}^{+}$. Therefore, changes of $\varphi$ are only attached to changes of $\mathbf{E}_{e}^{+}$, but not to changes of $\mathbf{E}_{e}^{-}$. The partition $\hat{\psi}_{e}$ defined in equation (59), has been taken from Miehe et al. [27].

The fracture energy $\hat{\psi}_{f}$ is given by the sum of two contributions, a homogeneous one and a gradient one. Whereas the former favors the local state $\varphi=0$, the later penalizes rapid spatial variation and smears out the transition zone in which $\varphi$ varies between 0 and 1 . Aside from the effects of unilateral damage and plasticity, this choice for the free-energy density appears in the 
context of the variational approximation of brittle fracture (c.f. Bourdin et al. [40]), which by its turn was based on the phase-field approximation of the Mumford-Shah potential introduced by Ambrosio and Tortorelli [41].

Since we are concerned with a rate-independent theory, we assume that the response functions $\hat{\beta}$ and $\hat{b}$ are identically equal to zero. Further, we assume:

$$
\begin{aligned}
& \hat{a}\left(\mathbf{E}_{e}, e, \varphi, \nabla \varphi\right)=\sqrt{\frac{2}{3}} \sigma_{y}, \\
& \hat{\alpha}\left(\mathbf{E}_{e}, e, \varphi, \nabla \varphi\right)=0,
\end{aligned}
$$

The aforementioned constitutive specialization results in the following expressions for $\mathbf{S}, f$ and $F$ :

$$
\begin{gathered}
\mathbf{S}=\left((1-\varphi)^{2}+k_{c}\right)\left(\lambda\left\langle\operatorname{tr} \mathbf{E}_{e}\right\rangle \mathbf{I}+2 \mu \mathbf{E}_{e}^{+}\right)+\left(-\lambda\left\langle-\operatorname{tr} \mathbf{E}_{e}\right\rangle \mathbf{I}+2 \mu \mathbf{E}_{e}^{-}\right) \\
F=2(1-\varphi) \hat{\psi}_{e}^{+}\left(\mathbf{E}_{\mathrm{e}}\right)-\frac{g_{f}}{l}\left(\varphi-l^{2} \triangle \varphi\right) \\
f=\left|\mathbf{S}_{0}\right|-\sqrt{\frac{2}{3}} \sigma_{y}\left(1+\sqrt{\frac{2}{3}} \frac{E e}{\sigma_{y}}\right)^{n}
\end{gathered}
$$

\section{Alternative handling of the phase-field equations}

The numerical treatment of the phase-field equations (56) is admittedly difficult. In the past, and within the context of gradient damage models, algorithms have been proposed to solve similar problems. Typically, we mention the algorithm proposed by Liebe et al. [42] which has been borrowed from nonlinear programming methods. This technique primarily consists of an iterative method which sequentially searches for the active sets, i.e the regions where $\dot{\varphi}<0$, and a posterior returning to the feasible domain defined by $\dot{\varphi} \geq 0$.

Alternatively to that kind of procedures, the solution proposed by Miehe et al. [27] is here adopted. It is next described.

Let us consider the compact history field function $\mathcal{H}(t)$ defined as follows:

$$
\mathcal{H}(t)=\max _{s} h(s), \quad s \in[0, t]
$$

where

$$
h(t):=\hat{\psi}_{e}^{+}\left(\mathbf{E}_{\mathrm{e}}, t\right)
$$

Then, instead of solving $\varphi(t)$ using the phase-field equations (56), with $F$ defined by (65), it is solved the equation:

$$
2(1-\varphi(t)) \mathcal{H}(t)-\frac{g_{f}}{l}\left(\varphi(t)-l^{2} \triangle \varphi(t)\right)=0,
$$

In Appendix I, and after introducing simplifying assumption, we sketch the equivalence between the phase-field equations (56) and (69).

Summary of governing equations: Table 1 summarizes the governing equations of the coupled rate-independent elasto-plastic phase-field model. 
Balance equations:

$$
\begin{gathered}
\operatorname{div} \mathbf{S}+\mathbf{b}=\mathbf{0} ; \quad \text { on } \mathcal{B} \quad \text { and } \quad \mathbf{S n}=\mathbf{s} \quad \text { on } \partial \mathcal{B}_{S} \\
2(1-\varphi) \mathcal{H}-\frac{g_{f}}{l}\left(\varphi-l^{2} \triangle \varphi\right)=0 ; \quad \text { on } \mathcal{B} \quad \text { and } \nabla \varphi \cdot \mathbf{n}=0 \quad \text { on } \partial \mathcal{B}_{\varphi}
\end{gathered}
$$

with:

$$
\begin{gathered}
\mathbf{S}=\left((1-\varphi)^{2}+k_{c}\right)\left(\lambda\left\langle\operatorname{tr} \mathbf{E}_{e}\right\rangle \mathbf{I}+2 \mu \mathbf{E}_{e}^{+}\right)+\left(-\lambda\left\langle-\operatorname{tr} \mathbf{E}_{e}\right\rangle \mathbf{I}+2 \mu \mathbf{E}_{e}^{-}\right) \\
\mathcal{H}(t)=\max h(s)=\max \hat{\psi}_{e}^{+}\left(\mathbf{E}_{\mathrm{e}}(s)\right), \quad s \in[0, t] \\
\dot{e} \geq 0, \quad f \leq 0, \quad \dot{e} f=0, \quad \gamma_{\mathrm{r}}=f ; \quad \text { on } \mathcal{B} ; \\
f=\left|\mathbf{S}_{0}\right|-\sqrt{\frac{2}{3}} \sigma_{y}\left(1+\sqrt{\frac{2}{3}} \frac{E e}{\sigma_{y}}\right)^{n}
\end{gathered}
$$

Table 1: Summary of the governing equations of the coupled elasto-plastic-phase field problem.

\section{Numerical model of the boundary value problem (BVP)}

In order to numerically solve the BVP defined in the previous section, the variational format presented in the principle of the virtual power (8) is reconsidered. Then, after defining admissible variations of the kinematical descriptors $(\tilde{\mathbf{u}}$ and $\tilde{\varphi})$, the corresponding variational equations are derived. In this format, the problem is amenable to be approached by means of a finite element technique. In the following, we consider a rate-independent constitutive model of fracture and plasticity.

Let us consider the body $\mathcal{B}$. Essential boundary conditions in displacements are prescribed on $\partial \mathcal{B}_{u}$, that is, $\mathbf{u}=\overline{\mathbf{u}}$ on $\partial \mathcal{B}_{u}$. Whereas tractions are prescribed on the boundaries $\partial \mathcal{B}_{S}$ and $\partial \mathcal{B}_{\xi}$. That is, $\mathbf{S n}=\mathbf{s}$ on $\partial \mathcal{B}_{S}$, and $\nabla \varphi \cdot \mathbf{n}=0$ on $\partial \mathcal{B}_{\xi}$. Initial condition are defined for $\varphi$. A possible choice is: $\varphi=0$ on $\mathcal{B}$.

Let us consider the following functional spaces defined on $\mathcal{B}$ :

$$
\begin{aligned}
& \mathcal{V}^{u}=\left\{\mathbf{u} \in \mathbf{H}^{1} \mid \mathbf{u}=\overline{\mathbf{u}} \quad \text { on } \quad \partial \mathcal{B}_{\mathbf{u}}\right\} \\
& \mathcal{V}^{\varphi}=\left\{\varphi \in \mathbf{H}^{1}\right\}
\end{aligned}
$$

with $\mathbf{H}^{1}$ being a space of smooth enough functions. $\mathcal{V}^{u}$ is the space of admissible displacement and $\mathcal{V}^{\varphi}$ the space of admissible phase-fields. Corresponding admissible spaces of variations are:

$$
\begin{aligned}
& \mathcal{V}_{0}^{u}=\left\{\mathbf{u} \in \mathbf{H}^{1} \mid \mathbf{u}=\mathbf{0} \quad \text { on } \quad \partial \mathcal{B}_{\mathbf{u}}\right\} \\
& \mathcal{V}_{0}^{\varphi}=\mathcal{V}^{\varphi}
\end{aligned}
$$

Then, the variational problem consists of finding the functions $\mathbf{u} \in \mathcal{V}^{u}$ and $\varphi \in \mathcal{V}^{\varphi}$ fulfilling the two following equations: 


$$
\begin{aligned}
& \int_{\mathcal{B}} \mathbf{S}(\mathbf{u}, e, \varphi) \cdot \tilde{\mathbf{E}} \mathrm{d} v-\int_{\mathcal{B}} \mathbf{b} \cdot \tilde{\mathbf{u}} \mathrm{d} v-\int_{\partial \mathcal{B}_{S}} \mathbf{s} \cdot \tilde{\mathbf{u}} \mathrm{d} a=0 ; \quad \forall \tilde{\mathbf{u}} \in \mathcal{V}_{0}^{u} \\
& \int_{\mathcal{B}} l_{f}(\nabla \varphi \cdot \nabla \tilde{\varphi}) \mathrm{d} v+\int_{\mathcal{B}}\left(-2(1-\varphi) \mathcal{H}(\mathbf{u}, e)+\frac{g_{f}}{l} \varphi\right) \tilde{\varphi} \mathrm{d} v=0 ; \quad \forall \tilde{\varphi} \in \mathcal{V}_{0}^{\varphi}
\end{aligned}
$$

jointly with the kinematics equations: (2)-(3), constitutive equations: (TI-3), (TI-4), (TI-5) and (TI-6).

\subsection{Finite element implementation}

A mixed finite element with equal order of interpolation for displacements $\mathbf{u}$ and phase-field $\varphi$ is proposed.

Let us consider the spatial discretization for each of these fields as follows:

$$
\begin{aligned}
& \mathbf{u}(\mathbf{x})=\sum_{i=1}^{n_{\text {node }}} N_{i}(\mathbf{x}) \mathbf{u}_{i} \\
& \varphi(\mathbf{x})=\sum_{i=1}^{n_{\text {node }}} N_{i}(\mathbf{x}) \varphi_{i}
\end{aligned}
$$

where $n_{\text {node }}$ stands for the number of nodes in the finite element mesh. The standard shape functions related to node $i$ is denoted $N_{i}(\mathbf{x})$. At the same node, displacement and phase-field interpolation parameters are denoted $\mathbf{u}_{i}$ and $\varphi_{i}$, respectively.

The corresponding spatial discretization of the virtual fields reads:

$$
\begin{aligned}
& \tilde{\mathbf{u}}(\mathbf{x})=\sum_{i=1}^{n_{\text {node }}} N_{i}(\mathbf{x}) \tilde{\mathbf{u}}_{i} \\
& \tilde{\varphi}(\mathbf{x})=\sum_{i=1}^{n_{\text {node }}} N_{i}(\mathbf{x}) \tilde{\varphi}_{i}
\end{aligned}
$$

where $\tilde{\mathbf{u}}_{i}$ and $\tilde{\varphi}_{i}$ are virtual variations of node $i$.

In a generic finite element $\mathfrak{e}$, the strain $\mathbf{E}$ and the admissible variation of strain $\tilde{\mathbf{E}}$ are given as follows:

$$
\mathbf{E}(\mathbf{x})=\mathbb{B}^{\mathfrak{e}}(\mathbf{x}) \mathbf{u}^{\mathfrak{e}} \quad \tilde{\mathbf{E}}(\mathbf{x})=\mathbb{B}^{\mathfrak{e}}(\mathbf{x}) \tilde{\mathbf{u}}^{\mathfrak{e}}
$$

where $\mathbf{u}^{\mathfrak{e}}$ is the nodal displacement vector of the element $\mathfrak{e}$. The strain-displacement matrix $\mathbb{B}^{\mathfrak{e}}$ is defined according with the B-Bar technique (c.f. Simo and Hughes [38]), i.e. quadrilateral finite elements with bilinear interpolation. Within a two-dimensional setting, the matrix $\mathbb{B}^{\mathfrak{e}}$ is:

$$
\begin{aligned}
& \mathbb{B}^{\mathfrak{e}}=\left[\begin{array}{llll}
\mathbf{B}_{1}^{\mathfrak{e}} & \mathbf{B}_{2}^{\mathfrak{e}} & \ldots, & \mathbf{B}_{n_{\text {node }}^{\mathfrak{e}}}^{\mathfrak{e}}
\end{array}\right] ;
\end{aligned}
$$

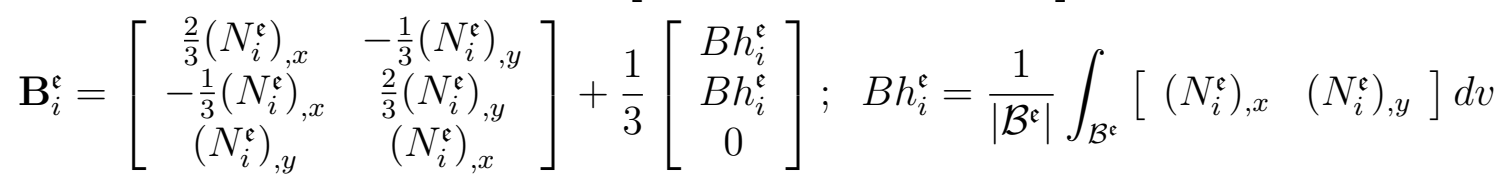


The gradient of the phase-field and the gradient of the admissible virtual variations are:

$$
\nabla \varphi(\mathbf{x})=\sum_{i=1}^{n_{\text {node }}}\left[\nabla N_{i}\right](\mathbf{x}) \varphi_{i} ; \quad \nabla \tilde{\varphi}(\mathbf{x})=\sum_{i=1}^{n_{\text {node }}}\left[\nabla N_{i}\right](\mathbf{x}) \tilde{\varphi}_{i} ; \quad\left[\nabla N_{i}\right]=\left[\begin{array}{c}
\left(N_{i}\right)_{, x} \\
\left(N_{i}\right)_{, y}
\end{array}\right]
$$

\subsubsection{Interpolation order selection of the fields in the mixed finite element}

In the context of mixed finite element approaches (interpolation of $\mathbf{u}$ and $\varphi$ ), and according to the analysis presented by Bourdin et al. [26], selection of low orders of interpolation for both fields is advantageous. However, it is known that equations coupling two fields may induce numerical locking. And locking effect tends to decrease with the use of high-order finite elements.

In fact, Verhoosel and de Borst [43] (c.f. also Simone [44]) claimed that numerical locking arises when the phase-field problem is solved with equal low order of interpolation (linear or bilinear) for $\mathbf{u}$ and $\varphi$. In this case, numerical locking induces stress field oscillations, which can be relieved with a displacement interpolation of third, or higher, order.

We have also observed stress oscillation in numerical solutions obtained with low order of interpolations. However, displacement and phase-field solutions are not affected. While, the stress oscillation can be alleviated through a standard smoothing technique without appealing to higherorder of interpolations.

Furthermore, our experience indicates that only a marginal increase of the error convergence rate, with respect to the finite element mesh size, is observed when increasing the interpolation order of the displacement field. Note that, in the case of elastic materials, convergence to sharp crack solutions requires both conditions: mesh size, as well as phase-field regularization parameter $l$, going to zero. Thus, only increasing the interpolation order does not necessarily implies increase of convergence rate. This observation agrees with that expressed by Bourdin et al. [26].

\subsubsection{Discrete equations of the quasi-static problem}

Substituting equations (74) - (80) into the variational problem (72) and (73), yields:

- Force balance:

$$
\bigwedge_{\mathfrak{e}=1}^{n_{\text {elem }}} \int_{\mathcal{B}^{\mathfrak{e}}}\left(\mathbb{B}^{\mathfrak{e}}\right)^{T} \mathbf{S}(\mathbf{u}, e, \varphi) d v-\mathbf{F}_{u}^{\mathrm{ext}}=\mathbf{0}
$$

where $\Lambda$ is the finite element assembling operator, $n_{\text {elem }}$ is the number of finite elements in the mesh and $\mathbf{S}$ is the stress term defined by the constitutive model. The vector $\mathbf{F}_{u}^{\text {ext }}$ is derived from the second and third terms in (72) and represents the conventional external forces. In expression (81), the variables $\mathbf{u}$ and $\varphi$ are the vector of nodal displacements and phase-field respectively, while $e$ is the vector collecting the accumulated plastic strains of all Gauss points.

- Damage equation: 


$$
\left[\mathbb{K}_{\varphi}+\mathbb{M}_{\varphi}\right][\varphi]-\mathbb{F}_{\varphi}^{\mathrm{ext}}=\mathbf{0}
$$

where:

$$
\begin{aligned}
\mathbb{K}_{\varphi}=\bigwedge_{\mathfrak{e}=1}^{n_{\text {elem }}} \mathbb{K}_{\varphi}^{\mathfrak{e}} ; & {\left[\mathbb{K}_{\varphi}^{\mathfrak{e}}\right]_{i, j}=\int_{\mathcal{B}^{\mathfrak{e}}} l g_{f}\left(\left(\nabla N_{i}^{\mathfrak{e}}\right)^{T} \cdot \nabla N_{j}^{\mathfrak{e}}\right) d v ; \quad\left[\nabla N_{i}^{\mathfrak{e}}\right]=\left[\begin{array}{c}
N_{i, x}^{\mathfrak{e}} \\
N_{i, y}^{\mathfrak{e}}
\end{array}\right] ; } \\
\mathbb{M}_{\varphi}=\bigwedge_{\mathfrak{e}=1}^{n_{\text {elem }}} \mathbb{M}_{\varphi}^{\mathfrak{e}} ; & {\left[\mathbb{M}_{\varphi}^{\mathfrak{e}}\right]_{i, j}=\int_{\mathcal{B}^{\mathfrak{e}}}\left(2 \mathcal{H}(\mathbf{u}, e)+\frac{g_{f}}{l}\right)\left(\left(N_{i}^{\mathfrak{e}}\right)^{T} \cdot N_{j}^{\mathfrak{e}}\right) d v ; } \\
\mathbb{F}_{\varphi}^{\text {ext }}=\bigwedge_{\mathfrak{e}=1}^{n_{\text {elem }}}\left(\mathbb{F}_{\varphi}^{\mathfrak{e}}\right)^{\text {ext }} ; & {\left[\left(\mathbb{F}_{\varphi}^{\mathfrak{e}}\right)^{\text {ext }}\right]_{i}=\int_{\mathcal{B}^{\mathfrak{e}}} N_{i}(2 \mathcal{H}(\mathbf{u}, e)) d v }
\end{aligned}
$$

\subsubsection{Time integration scheme}

Let us denote $\mathbf{R}_{\mathbf{S}}(\mathbf{u}, e, \varphi)$ and $\mathbf{R}_{\varphi}(\mathbf{u}, e, \varphi)$ the residues of equations (81) and (82), respectively. Then, these equations can be rewritten in short as follows:

$$
\begin{aligned}
& \mathbf{R}_{\mathbf{S}}(\mathbf{u}, e, \varphi)=\mathbf{0} \\
& \mathbf{R}_{\varphi}(\mathbf{u}, e, \varphi)=\mathbf{0}
\end{aligned}
$$

where the implicit dependence of the fields with the pseudo-time $t$ is understood.

Equations (84) can be integrated by appealing to the staggered schema shown in Table 2. It consists of solving successively equations $(84)_{1}$ and $(84)_{2}$. First, equation $(84)_{1}$ is solved to obtain $\mathbf{u}_{n+1}^{1}$ and $e_{n+1}^{1}$ with $\varphi_{n}$ fixed. Subindices and supraindices denoting the integration time step and subiteration, respectively. In a second step, equation $(84)_{2}$ is solved to obtain $\varphi_{n+1}^{1}$ with $\mathbf{u}_{n+1}^{1}$ and $e_{n+1}^{1}$ fixed. Finally, a sub-iteration (mentioned as point 3 in Table 2) can be carried out, which consists of a sequential repetition of the first and second steps, in order to reach a converged solution.

By solving the equation (TII-1), in the first step of this staggered scheme, the incremental inequality $\Delta e \geq 0$ is pointwise guaranteed by means of a conventional return mapping algorithm.

Also, by solving the equation (TII-2) in the second step of the staggered scheme, the inequality $\Delta \varphi(\mathbf{x})=\varphi_{n+1}(\mathbf{x})-\varphi_{n}(\mathbf{x}) \geq 0$ in $\mathcal{B}$ is satisfied (c.f. Miehe et al. [27], see also Appendix I) by defining the compact history field $\mathcal{H}\left(\mathbf{E}_{e}, t_{n+1}\right)$ as follows:

$$
\begin{array}{cll}
\mathcal{H}\left(\mathbf{x}, t_{n+1}\right)=\hat{\psi}_{e}^{+}\left(\mathbf{E}_{e}\left(\mathbf{x}, t_{n+1}\right)\right) & \text { if } & \hat{\psi}_{e}^{+}\left(\mathbf{E}_{e}\left(\mathbf{x}, t_{n+1}\right)\right)>\hat{\psi}_{e}^{+}\left(\mathbf{E}_{e}\left(\mathbf{x}, t_{n}\right)\right) \\
\mathcal{H}\left(\mathbf{x}, t_{n+1}\right)=\mathcal{H}\left(\mathbf{x}, t_{n}\right) & \text { if } & \hat{\psi}_{e}^{+}\left(\mathbf{E}_{e}\left(\mathbf{x}, t_{n+1}\right)\right) \leq \hat{\psi}_{e}^{+}\left(\mathbf{E}_{e}\left(\mathbf{x}, t_{n}\right)\right)
\end{array}
$$

Thus, the function $\mathcal{H}$ satisfies:

$$
\mathcal{H}\left(\mathbf{x}, t_{n+1}\right) \geq \mathcal{H}\left(\mathbf{x}, t_{n}\right)
$$

for every time in $\mathcal{B}$.

As can be seen in the next Section, we show that using this technique, the inequality $\Delta \varphi(\mathbf{x}) \geq 0$ is satisfied in the analyzed points. 
LOOP over time steps: $(n+1)$

Given $\mathbf{u}_{n}, e_{n}, \varphi_{n}$ and $\left(\mathbf{F}_{u}^{e x t}\right)_{n+1},\left(\mathbb{F}_{\varphi}^{e x t}\right)_{n+1}$;

Find: $\mathbf{u}_{n+1}, e_{n+1}$ and $\varphi_{n+1}$ as follows:

iter $=1 \quad ; \quad \mathbf{u}_{n+1}^{0}=\mathbf{u}_{n} ; \quad e_{n+1}^{0}=e_{n} ;$

1) Using a Newton-Raphson iteration, find $\mathbf{u}_{n+1}^{i t e r}$ and $e_{n+1}^{i t e r}$ by solving:

$$
\mathbf{R}_{\mathbf{S}}\left(\mathbf{u}_{n+1}^{i t e r}, e_{n+1}^{i t e r}, \varphi_{n}\right)=\mathbf{0} ;
$$

2) Find $\varphi_{n+1}^{i t e r}$, with $\mathbf{u}_{n+1}^{i t e r}$ fixed, by solving the system of linear equations:

$$
\mathbf{R}_{\varphi}\left(\mathbf{u}_{n+1}^{i t e r}, e_{n+1}^{i t e r}, \varphi_{n+1}^{i t e r}\right)=\mathbf{0}
$$

3) if $\left\|\varphi_{n+1}^{i t e r}-\varphi_{n}\right\|>0.1$, then:

DO WHILE $\left\|\mathbf{u}_{n+1}^{i t e r}-\mathbf{u}_{n+1}^{i t e r-1}\right\| /\left\|\mathbf{u}_{n+1}^{i t e r}\right\|>$ tol $\quad \& \quad$ iter $\leq$ MAXiter iter $=$ iter +1 ;

Use a fixed-point iteration to find $\mathbf{u}_{n+1}^{i t e r}, e_{n+1}^{i t e r}$ and $\varphi_{n+1}^{i t e r}$ by iteratively solving the sequence of steps 1) and 2) (equations (TII-1) and (TII-2)); In equations (TII-1), $\varphi_{n}$ should be replaced by $\varphi_{n+1}^{i t e r-1}$

END DO

Assign: $\mathbf{u}_{n+1}=\mathbf{u}_{n+1}^{i t e r} ; e_{n+1}=e_{n+1}^{i t e r} ; \varphi_{n+1}=\varphi_{n+1}^{i t e r}$;

END LOOP over time steps

Table 2: Staggered time integration scheme for solving the coupled elasto-plastic-phase field problem. 


\section{Numerical model assessment}

The numerical assessment of the model is carried out by simulating two kinds of tests. First, in sub-Section 4.1, a series of standard linear elastic fracture mechanic problems (LEFM) are solved. An accurate estimation of the expended external energy to achieve complete structural failure is evaluated. As a consequence of considering linear elastic response, the accuracy of the numerical solutions can be easily sized up by comparing the expended external energies with the analytical dissipated energies governed by the parameter $g_{f}$. Also, an identical comparison evidences the low sensitivity of the expended external energy with the length parameter $l$.

In the second part, sub-Section 4.2, the phase-field approach is studied in combination with an elasto-plastic model. The propagation of a pre-existent crack in Mode I under the small scaleyielding assumption is simulated. A detailed analysis is accomplished about the sensitivity of the material parameters; specifically the relationship between the parameters of the plastic and phasefield models. A discussion of results is presented. With the present approach, it is concluded that the regularization parameter of the phase-field model, $l$, should be reinterpreted in order to capture the interaction effects between both mechanisms: plasticity and damage. This parameter must not go to zero. Instead, it has to be considered as a material characteristic length, of finite size, which should be characterized jointly with the fracture energy.

All solutions in this Section have been obtained assuming plane strain hypothesis.

\subsection{Linear elastic fracture problems}

The following numerical assessments of the phase-field approach are carried out by using a set of linear elastic fracture mechanic (LEFM) problems:

i) in Sub-Section 4.1.1-case 1, the phase-field model is validated by comparing results available in the literature;

ii) in Sub-Section 4.1.1-case 2, the convergence properties of the staggered time-integration algorithm, proposed in Table 2, is studied.

In both cases (1 and 2) of Sub-Section 4.1.1, several Single Edged Notched Tension (SENT) tests, undergoing uniform stresses at both edges, are simulated.

iii) in Sub-Sections 4.1.2 and 4.1.3, two specimens are simulated till reaching the complete loss of the structural loading carrying capacity. In both cases, the dissipated energies are evaluated and compared with the exact ones obtained in closed form.

These findings provide a better understanding of the results evaluated in the second part (SubSection 4.2) of this Section.

\subsubsection{SENT test}

Single Edged Notched Tension (SENT) tests under uniform tractions at the upper and lower edges, as shown in Figure 1-a, are numerically solved using a phase-field approach with the assumption of linear elastic response in the bulk material. Classical analytical solutions for this problem are widely reported in standard books of fracture mechanics. 


\section{Case 1: Load estimation at the crack propagation onset}

Figure 1 shows the geometry of the specimen and the finite element model that is used to estimate the external load which initiates the crack propagation process. Due to symmetry conditions, only one half of the specimen (the upper part) is modeled. A finite element mesh with bilinear quadrilateral elements is used. Note that close to the crack propagation zone, a structured mesh is adopted with elements of size: $\left(h^{\mathfrak{e}} \times h^{\mathfrak{e}}\right)$ being $h^{\mathfrak{e}}=0.3125[\mathrm{~mm}]$.

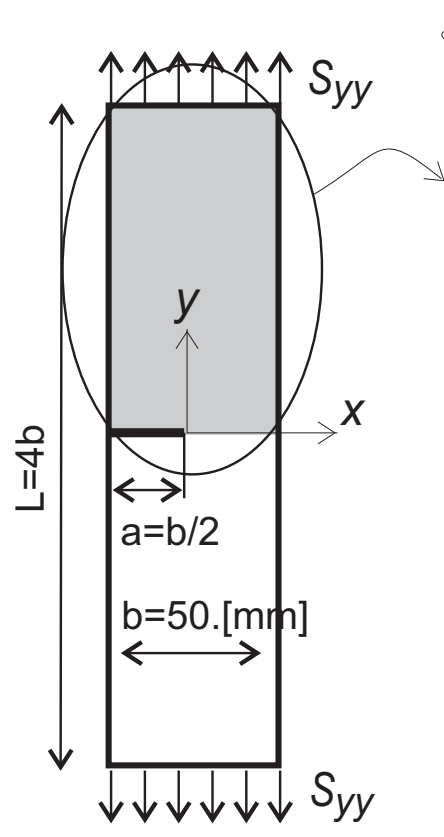

(a)

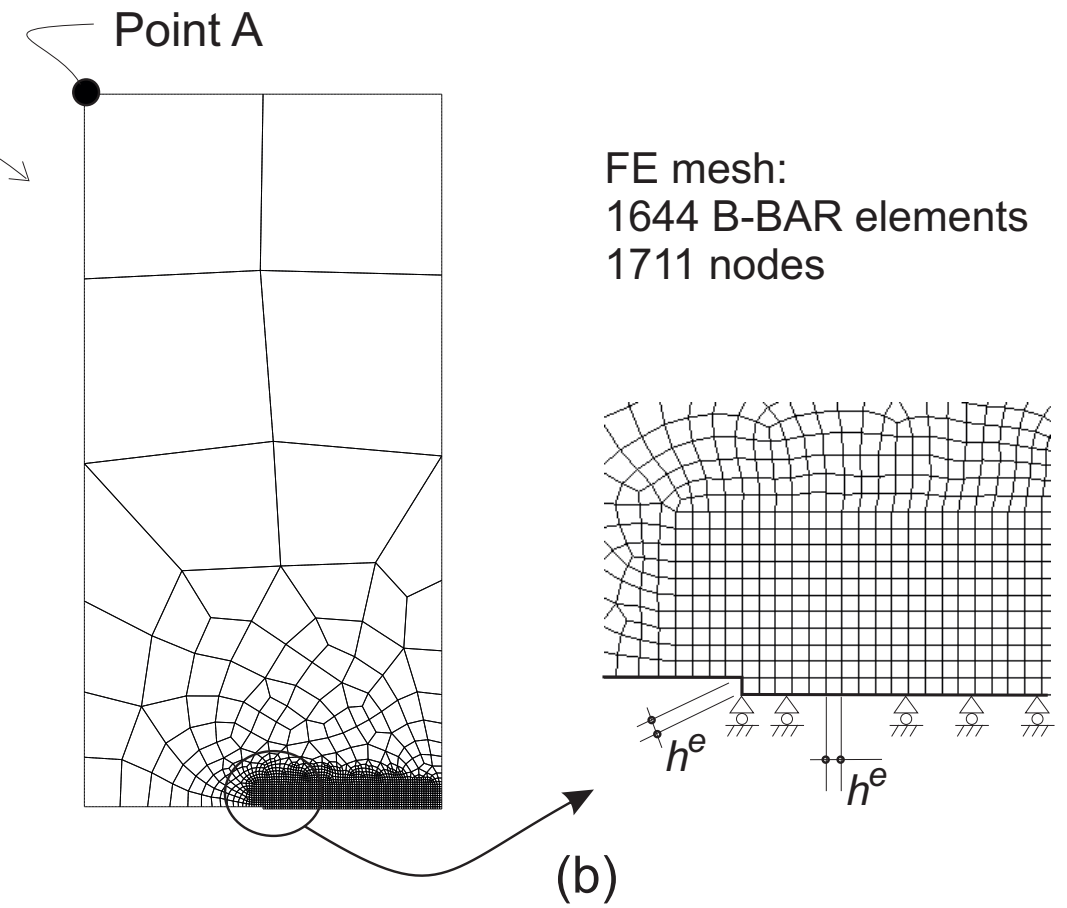

(b)

Figure 1: SENT test: geometrical data and mechanical model.

The stress intensity factor at the crack propagation onset for the SENT test, as a function of the fracture energy, is reported in the book of Gross and Seelig, 2006. From this reference work, it can be determined the corresponding critical uniform stress $\left(S_{y y}^{c r i t}\right)$ acting on the upper and lower specimen edges which initiates the crack propagation process. In the present numerical solutions, we adopt the crack propagation onset as the time instant when the bottom node in the notch root reaches a damage value: $\varphi>0.97$.

For the geometry depicted in Figure1-a, the critical stress is given by:

$$
S_{y y}^{c r i t}=\frac{1}{B} \sqrt{\frac{E}{1-\nu^{2}} \frac{g_{f}}{2 b}} \quad ; \quad B=\frac{1.762+0.37\left(1-\sin \left(\frac{\pi}{4}\right)\right)^{3}}{\cos \left(\frac{\pi}{4}\right)}
$$

Figure 2-a plots the function: $S_{y y}^{c r i t}\left(g_{f}\right)$ after considering a Young's modulus: $E=5.5[G P a]$ and a Poisson ratios: $\nu=0.25$. In the same Figure, the finite element numerical solutions are depicted for three fracture energies: $g_{f}^{1}=1[\mathrm{~N} / \mathrm{mm}], g_{f}^{2}=5[\mathrm{~N} / \mathrm{mm}]$ and $g_{f}^{3}=10[\mathrm{~N} / \mathrm{mm}]$, respectively. The length parameter $l$ of the phase-field model is defined as: $l=1.2[\mathrm{~mm}]\left(\approx 4 h^{\mathfrak{e}}\right)$, and $k_{c}=10^{-8}$.

As can be observed in this Figure, very accurate numerical solutions for estimating the critical stresses are obtained. A similar conclusion has been reported by Kuhn and Müller [45]. 
Figure 2-b plots the loads (per unit of thickness) versus the vertical displacement of the point A (depicted in Figure 1-b). The critical loads, at the crack propagation onset, are lower than the structural limit loads. Also, the structural response displays a slight non-linearity before reaching the limit load. Both effects suggest an inaccurate response obtained for this kind of problem, confirming that bilinear finite elements have deficiencies to resolve the sharp-crack linear elastic problem.

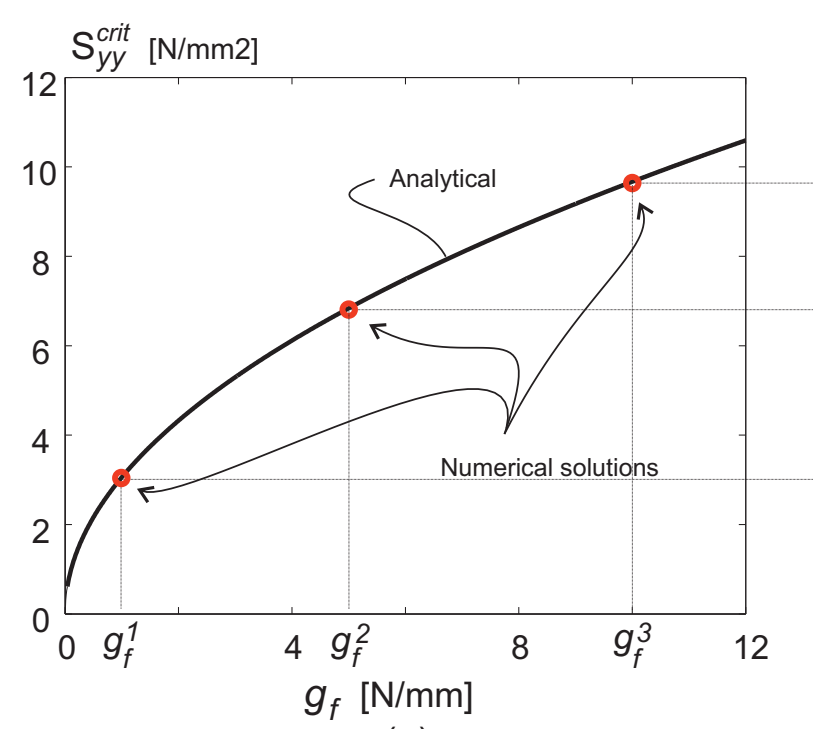

(a)

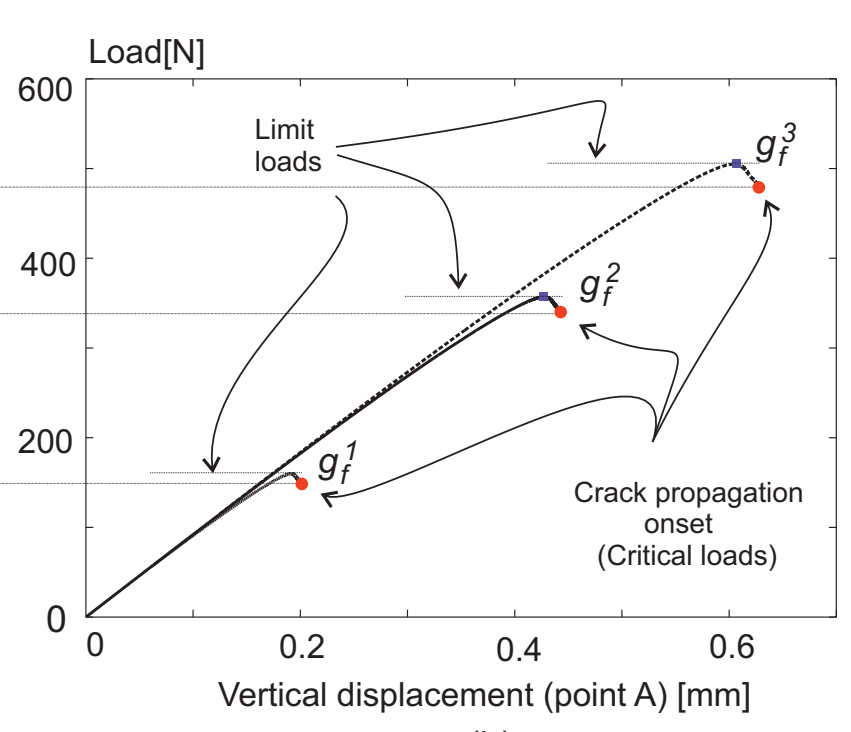

(b)

Figure 2: SENT tests undergoing uniform tractions on the top and bottom edges of the specimen (fracture energies: $\left.g_{f}^{1}=1 .[\mathrm{N} / \mathrm{mm}] ; g_{f}^{2}=5 .[\mathrm{N} / \mathrm{mm}] ; g_{f}^{3}=10 .[\mathrm{N} / \mathrm{mm}]\right)$. a) Critical average stresses $\left(S_{y y}^{c r i t}\right)$ vs. $g_{f}$, comparison between analytical and numerical results. b) Total load (per unit of thickness) vs. vertical displacement of Point A.

\section{Case 2: analysis of the integration scheme}

The item (3) in Table 2 describes a sub-iteration procedure which increases the time integration accuracy. The effects on including such sub-iteration procedure in the integration scheme is next evaluated. The analysis consist of comparing the computational cost versus the solution accuracy obtained in different instances: using, or not, the sub-iteration procedure.

The simulated SENT specimen is depicted in Figure 3-a. The elastic material is characterized with a Young's modulus $E=50.5[\mathrm{GPa}]$, Poisson ratio $\nu=0.40$, fracture energy $g_{f}=$ $0.5939[\mathrm{~N} / \mathrm{m}]$ and length parameter of phase-field model $l=0.05[\mathrm{~mm}]$. One half of the specimen is modeled with 6679 bilinear quadrilateral finite elements. A structured mesh is used in the region close to the crack tip, with the finite element size: $h^{\mathfrak{e}}=0.01[\mathrm{~mm}]$.

In Figure 3-b, the total load $(\mathrm{F})$ per unit of thickness vs. the vertical displacement of point $\mathrm{A}$ is plotted. This point is located at $2.5[\mathrm{~mm}]$ of the upper left corner of the specimen. When this displacement is considered, the equilibrium path displays a very marked snap-back behavior. Thus, in order to trace this response, the vertical displacement of the point B (depicted in Figure 3-a and close to the notch tip) is controlled through the parameter $s$. Figure 3-c displays the deformed specimen when the control parameter $s$ has reached the value $s=0.1[\mathrm{~mm}]$.

Figure 3-d plots three curves representing numerical errors versus the CPU time required to 
find the solution. Curves in Figure 3-d correspond to $M A X i t e r=1,2$ and 3, respectively. The parameter $M A X$ iter defines the number of sub-iterations performed during the time integration step, see Table 2. The integration scheme does not use sub-iterations when $M$ AXiter $=1$. Each point of the curves is obtained by modifying the value of the control parameter $\Delta s$. Note that, the smaller is $\Delta s$, the lower is the integration error and the larger is the computational time. All structural solutions have finished once the control parameter reached the value $s=\sum \Delta s=$ $0.1[\mathrm{~mm}]$.

The vertical axis of the plots in Figure 3-d corresponds to the logarithmic errors:

$$
\log (\text { error })=\log \left(\frac{W^{\text {ext }}-W_{E}^{\text {ext }}}{W_{E}^{\text {ext }}}\right)
$$

which is evaluated as the difference between the total external work (per unit of thickness):

$$
W^{\mathrm{ext}}=\int\left(\int_{\Delta_{s=0}}^{\Delta_{s=0.0001}} S_{y y} d u(s, x)\right) d x
$$

and the exact external work of comparison, $W_{E}^{\text {ext }}$. In expression (88), $S_{y y}$ and $d u(x)$ are the uniform stretching stresses and incremental displacements (which increase with the control parameter $s$ ) in the upper edge of the specimen. The x-integration is performed along this edge. While, the exact external work of comparison $W_{E}^{\text {ext }}$ is evaluated using a numerical solution obtained with an extremely small increment of the control parameter: $d s=10^{-5}[\mathrm{~mm}]$ and $M$ AXiter $=3$. In this case, the total CPU time is 108000. [sec].

From the plots in Figure 3-d, it can be concluded that the better performance of the integration scheme for identical CPU times is obtained with two sub-iterations per step: $M A X i t e r=3$.

\subsubsection{DENT panel test under uniform displacement}

In this sub-Section, the numerical solution accuracy for capturing the structural dissipated energy during a crack propagation problem is studied. A Double-edged notched test (DENT) is solved assuming linear elastic response. The geometry of the specimen is depicted in Figure 4. Uniform vertical displacements in the top and bottom edges of the specimen are prescribed.

A symmetric solution of the problem is simulated. Therefore, only one fourth of the specimen is modeled. A region in the specimen middle, of height equal to $3 .[\mathrm{mm}]$, is modeled with a structured and uniform finite element mesh (see Figure 4-b), with element sizes $1.67 h^{\mathfrak{e}} \times h^{\mathfrak{e}}$. Two meshes are designed with this criteria: the Mesh 1 has a finite element size: $h^{\mathfrak{e}}=0.1[\mathrm{~mm}]$, and the Mesh 2 has a size $h^{\mathfrak{e}}=0.3[\mathrm{~mm}]$. The material parameters are: Young's modulus $E=2.5[G P a]$, Poisson ratio $\nu=0.25$, fracture energy $g_{f}=5[\mathrm{~N} / \mathrm{mm}]$ and $k_{c}=10^{-9}$. In general, structural dissipated energies are influenced by $k_{c}$ when $k_{c}>10^{-7}$.

Figure 5-a depicts three plots that show total loads (per unit of thickness) vs. upper edge vertical displacements. Two of these curves have been obtained with Mesh $1(l=1.2[\mathrm{~mm}]$ and $l=2.4[\mathrm{~mm}])$ and the third one with Mesh $2(l=1.2[\mathrm{~mm}])$.

A load control scheme has been adopted to obtain these equilibrium curves consisting of the following procedure. Initially, the list of nodes located just above the crack path is collected (the direction of the crack path has been estimated beforehand and is the horizontal line coinciding with the bottom edge of the finite element model. Then, in every time step and from that list, it is 

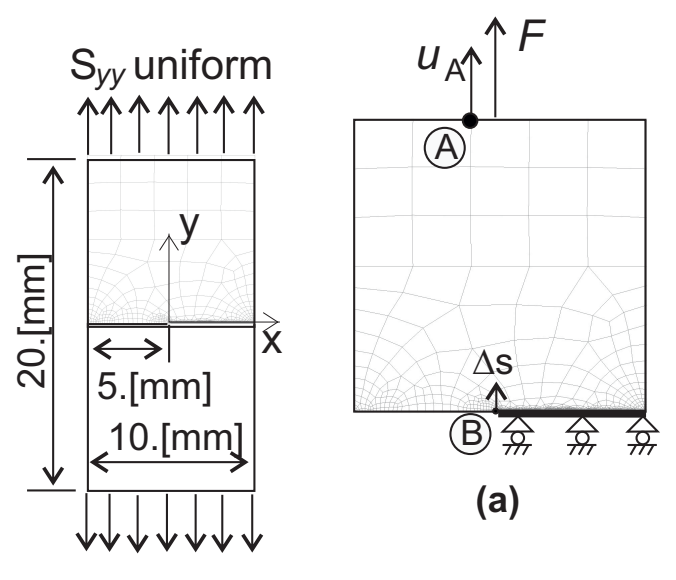

(a)

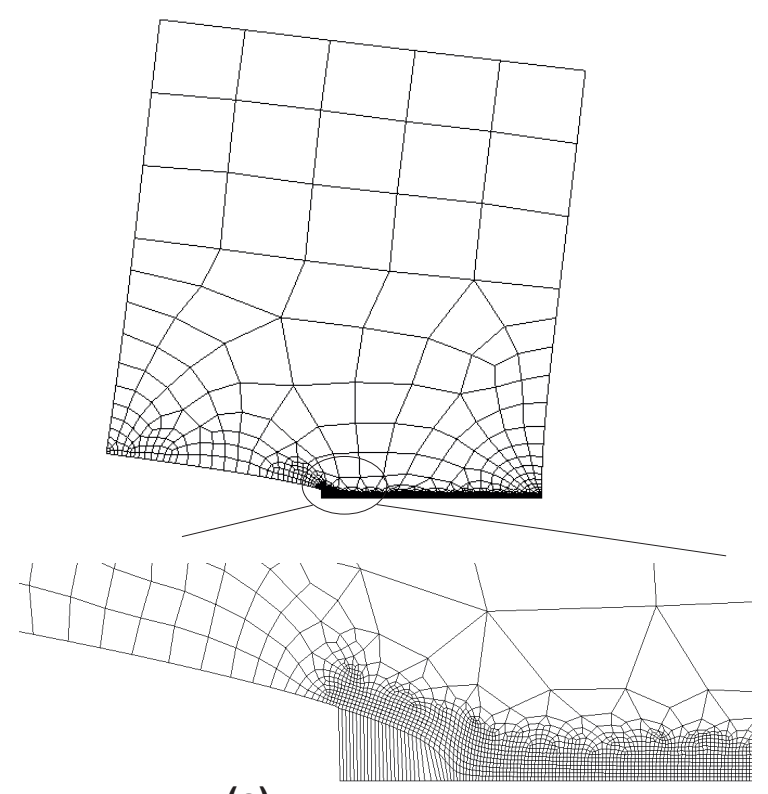

(c)

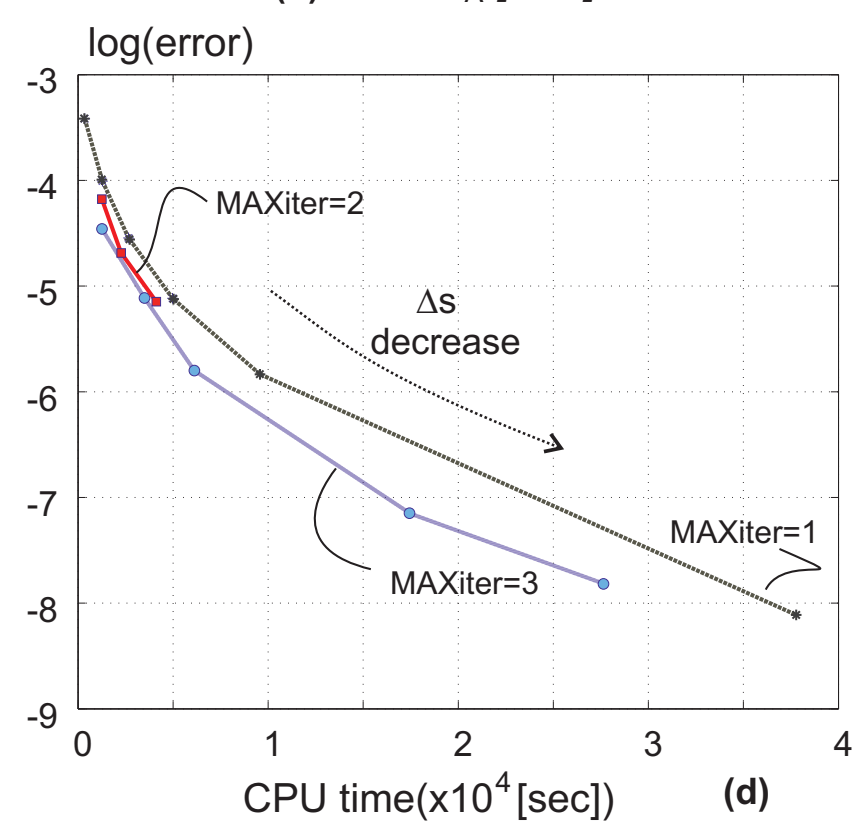

Figure 3: SENT tests undergoing uniform tractions. a) Specimen geometry and finite element mesh. b) Load (per unit thickness) vs. vertical displacement of point A. c) Deformed mesh at the end of analysis. d) Integration error vs. CPU time. 


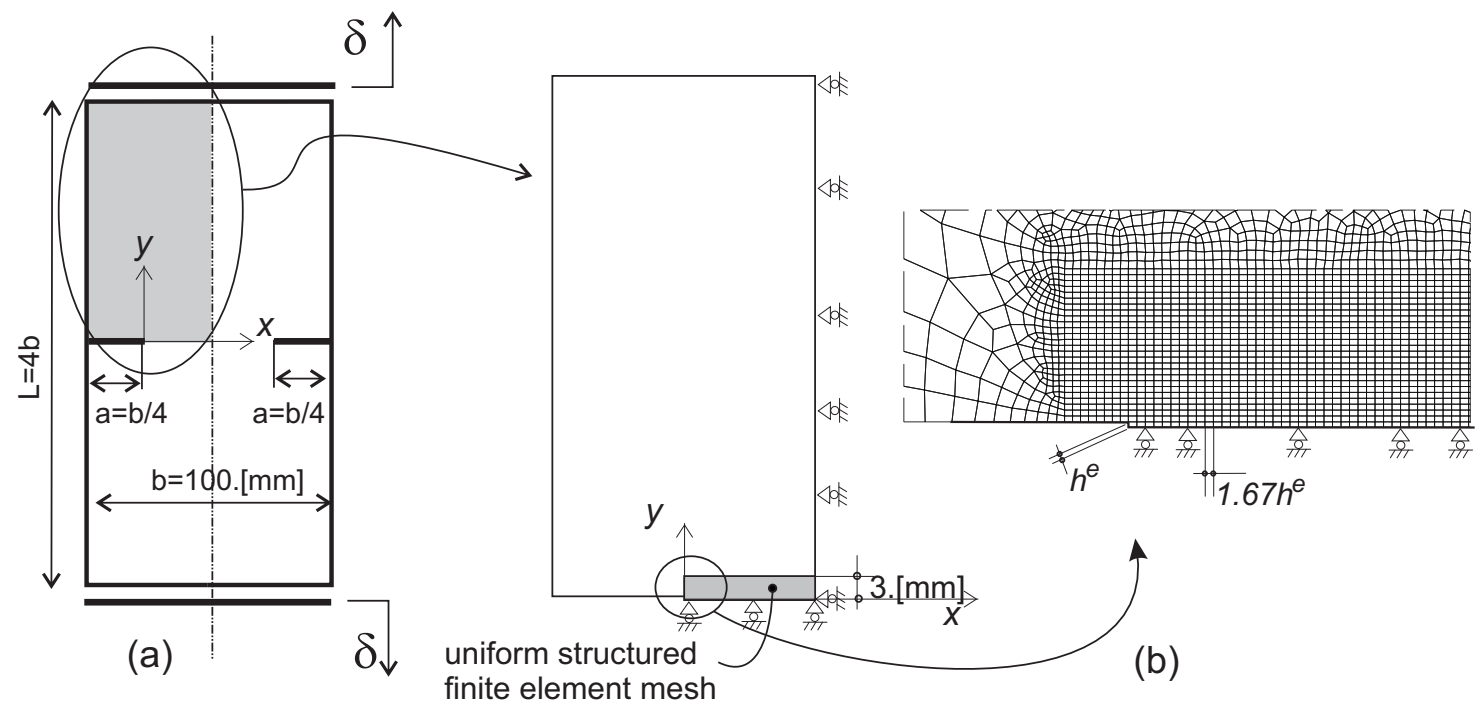

Figure 4: DENT panel test undergoing uniform displacements in the top and bottom edges. a) Geometrical data. b) Mechanical model and finite element mesh in the crack propagation region.

selected the node that has shown the maximum incremental vertical displacement. In the following time step, a displacement increment $\Delta s$ is prescribed in that node.

This test provides a clue about the capability of the numerical methodology to capture the total energy dissipated during the structural failure process. Due to the elastic bulk response, and considering a unit specimen thickness, the exact total dissipated energy for the symmetric solution is $g_{f}(b-2 a)=125[\mathrm{Nmm}]$. With the present numerical model, only the half of this energy $(62.5[\mathrm{Nmm}])$ is estimated because the upper part of the specimen is simulated.

The areas enclosed by the plots in Figure 5 represent half of the external work expended to produce the complete structural failure. Due to the elastic response of the material, these quantities are equivalent to the energy $D$ dissipated by the fracture propagation process across the specimen. Table 3 shows the expended external work obtained with both meshes (Mesh 1 and Mesh 2) and three different length parameters $(l=1.2[\mathrm{~mm}], l=1.8[\mathrm{~mm}]$ and $l=2.4[\mathrm{~mm}])$. The figures in parenthesis give the overestimation (in percent) of the numerically evaluated external work respect to the exact value that is expected from the crack propagation process $(62.5[\mathrm{Nmm}])$. Observe the slow convergence rate obtained with mesh refinement. Once again, this poor response evidences the difficulty that bilinear finite elements have in order to resolve the sharp-crack limit solution.

Additional discussion of these results are presented in the next sub-Section.

\begin{tabular}{|c|c|c|c|}
\hline & $l=1.2[\mathrm{~mm}]$ & $l=1.8[\mathrm{~mm}]$ & $l=2.4[\mathrm{~mm}]$ \\
\hline Mesh 1 & $72.5[\mathrm{Nmm}]$ & $72.7[\mathrm{Nmm}]$ & $73.0[\mathrm{Nmm}]$ \\
$h^{\mathfrak{e}}=0.1[\mathrm{~mm}]$ & $(16 \%)$ & $(16 \%)$ & $(16 \%)$ \\
\hline Mesh 2 & $83.0[\mathrm{Nmm}]$ & $78.6[\mathrm{Nmm}]$ & $77.3[\mathrm{Nmm}]$ \\
$h^{\mathfrak{e}}=0.3[\mathrm{~mm}]$ & $(32 \%)$ & $(25 \%)$ & $(23 \%)$ \\
\hline \hline
\end{tabular}

Table 3: DENT panel test under uniform displacement. External expended work to produce the complete structural failure.

Figure 6 displays the damage distribution along the vertical line, close to the initial crack tip, which is denoted y-y' (see the insert in Figure 6). The results corresponds to Mesh $1, l=2.4[\mathrm{~mm}]$. 


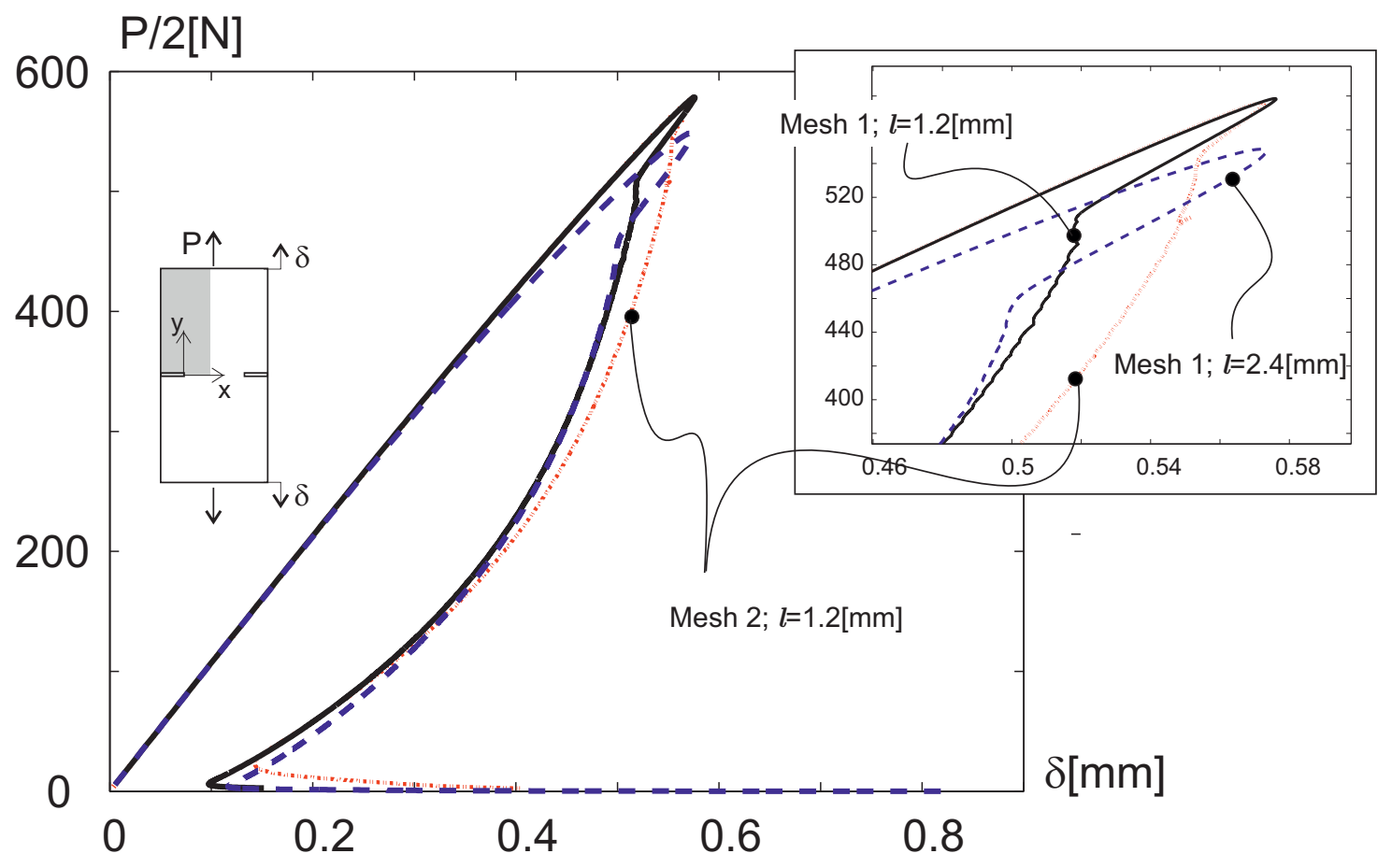

Figure 5: DENT panel test under uniform displacement: structural loads vs displacement curves. Solutions obtained with Mesh $1, l=1.2[\mathrm{~mm}]$ and $2.4[\mathrm{~mm}]$ and Mesh 2, $l=1.2[\mathrm{~mm}]$.

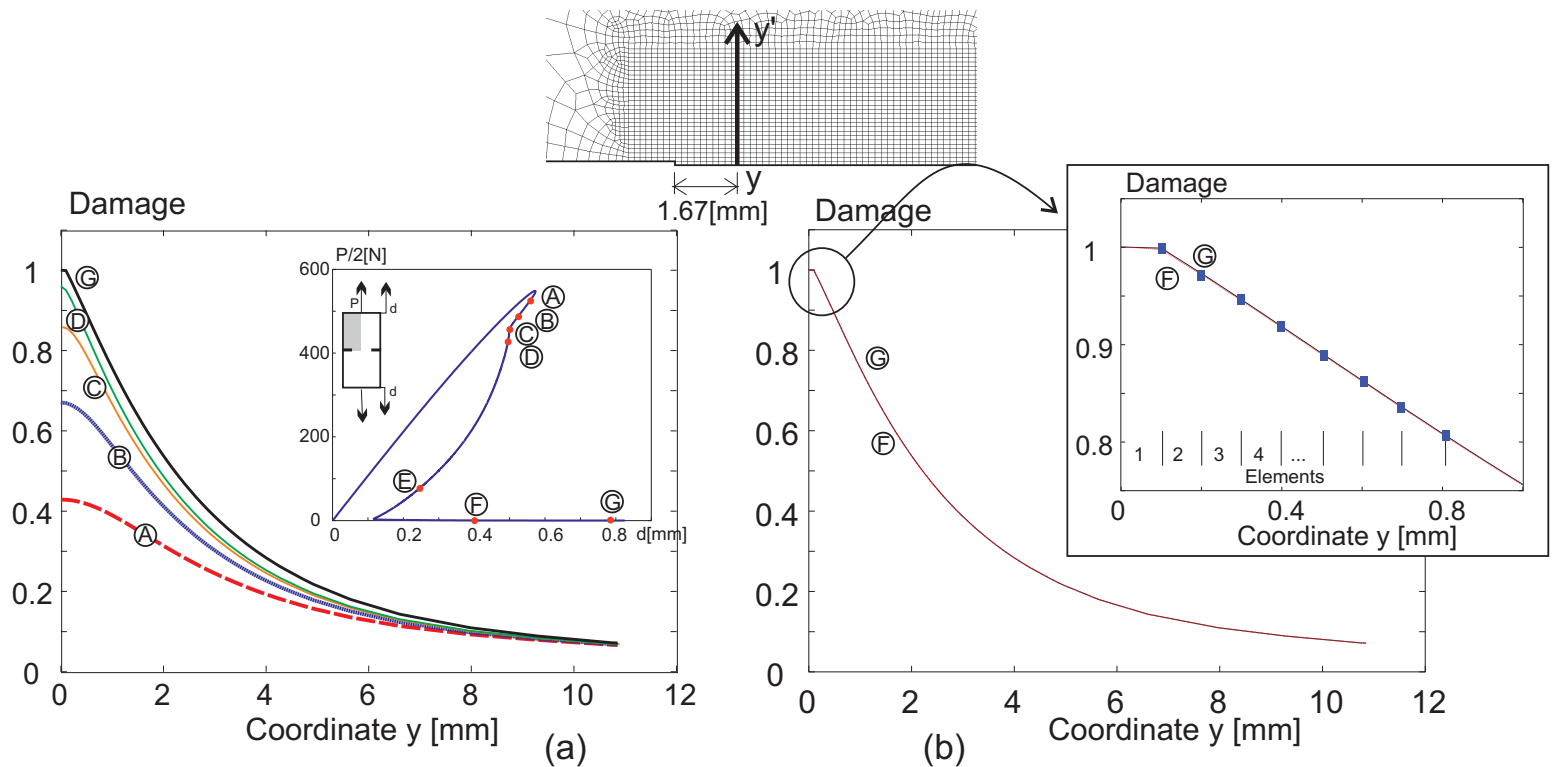

Figure 6: DENT panel test under uniform displacement. (a) Damage distribution along the vertical line y-y' (Mesh 1, $l=2.4[\mathrm{~mm}])$. Each curve corresponds to a loading stage that is marked in the structural plot inserted in the Figure. (b) Damage distribution at stages F and G, once the specimen has completely lost its structural capacity. 
Several plots displaying the evolution of damage are depicted in this Figure. Each plot corresponds to a different load stage which are marked with points in the structural response plot inserted in Figure 6-a.

In the plots of Figure 6-a, it can be observed that, as the structural loading proceeds, the damage evolution displays a monotonous increase: $\Delta \varphi(\mathbf{x}) \geq 0$, satisfying the phase-field model constraint: $\dot{\varphi} \approx \Delta \varphi(\mathrm{x}) \geq 0$. This property is observed for every point $\mathrm{x}$ along the line $\mathrm{y}-\mathrm{y}^{\prime}$ and for every time.

Figure 6-b shows additionally a very important feature which is characteristic of the phasefield model. The damage distributions along the vertical line $y-y$ ' in stages $F$ and $G$ are plotted in Figure 6-b. Both results have been taken from a completely degraded specimen while the crack is opening. The plots are almost indistinguishable. Therefore, although the crack opens continuously and strains tend to infinity in the localization zone, damage stops to progress in its vicinity. This conclusion, jointly with the response observed in the deformed specimens of Figures 3-c, 7-b and 9-a, allow us to conclude that the phase-field model correctly represents strain localization bands leading to strong discontinuity solutions.

This feature, which is additionally discussed in the following Sections, is not shared by some damage gradient models such as that presented in Peerlings et al. [46] (see additional details in Appendix II).
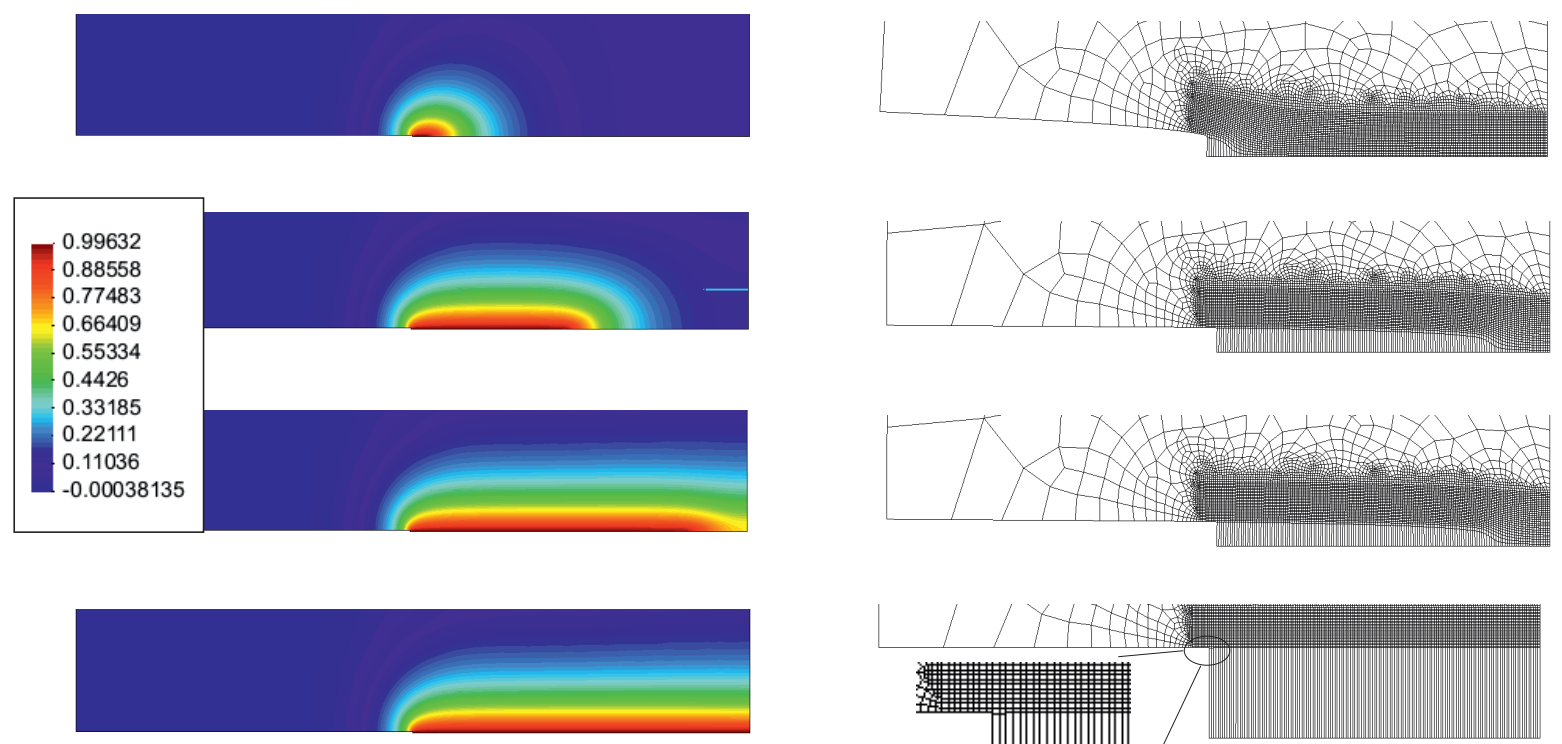

(a)

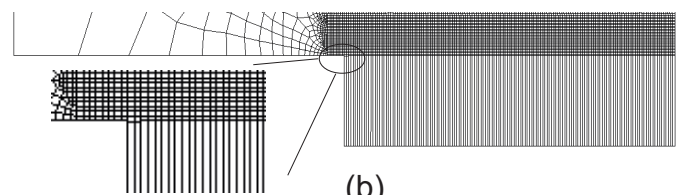

(b)

Figure 7: DENT panel test under uniform displacement. (a) Damage distribution (Mesh 1, $l=2.4[\mathrm{~mm}]$. (b) Deformed meshes. (Figures are depicted from top to bottom for the following vertical displacement at the upper edge: $0.20[\mathrm{~mm}]$, $0.44[\mathrm{~mm}], 0.50[\mathrm{~mm}]$ and $0.82[\mathrm{~mm}]$, respectively.

Figure 7-a shows a series of pictures displaying the distribution of damage at different loading stages. Also, Figure 7-b shows the deformed meshes at the same stages. Again, the last picture in Figure 7-b reflects the capability of the model to capture a strong discontinuity solution. 


\subsubsection{Single-Edge Notched Bending (SENB) test}

The SENB test displayed in Figure 8 is simulated and the total structural dissipated energy is analyzed.

Identical material parameters described in the previous Sub-Section 4.1.2 are adopted: Young's modulus $E=2.5[G P a]$, Poisson ratio $\nu=0.25$ and fracture energy $g_{f}=5 .[\mathrm{N} / \mathrm{mm}]$. Due to the problem symmetry, one half of the beam is modeled. The specimen geometry is depicted in Figure 8. The beam sizes are such that the analyzed domain is identical to that defined for the SENT test in the previous Sub-Section 4.1.2. Then, absolute finite element sizes and phase-field characteristic lengths are comparable in both tests (SENT and SENB). Similar to previous cases, the size of the notch is given by $h^{\mathfrak{e}}$.



(a)

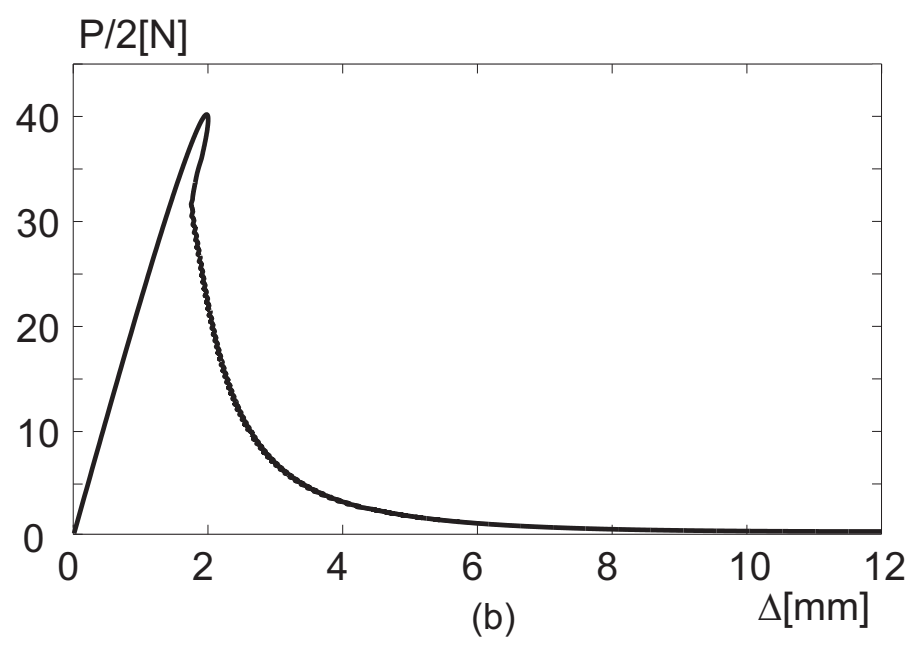

(b)

Figure 8: SENB test. (a) Geometry. (b) Load. vs. displacement curve.

The results have been obtained with three meshes. They have been designed with the same mesh refinement criteria as that adopted in Sub-Section 4.1.2. Mesh 1 and Mesh 2 coincide with the previous ones defined there (finite element sizes $h^{\mathfrak{e}}=0.1[\mathrm{~mm}]$ and $h^{\mathfrak{e}}=0.3[\mathrm{~mm}]$, respectively, in the region close to the crack tip), and Mesh A has finite element sizes $h^{\mathfrak{e}}=0.05[\mathrm{~mm}]$ in the same region.

Figure 8-b shows half of the load vs. vertical displacement of the load application point. The crack opening is controlled during the loading process imposing a similar strategy to that defined for the previous SENT test.

Figure 9-a depicts the deformed mesh and Figure 9-b the distribution of damage. Both results have been obtained at the end of analysis. Again in this case and once the material completely loses the loading carrying capacity, the deformed mesh shows a solution being compatible with a strong discontinuity kinematics.

Figure 10 depicts the distribution of damage. Only values having $\varphi \leq 0.01$ are shown. Figure 10 -a corresponds to the SENB test with $l=2.4[\mathrm{~mm}]$, at the initial stage of analysis, while Figure $10-\mathrm{b}$ displays the solution at the end of analysis. Figure 10-c plots the $\varphi$-distribution, at the initial stage, when the Poisson's ratio $\nu=0$. Comparing Figures 10 -a and $\mathrm{c}$ in the zone we have denoted with $\mathrm{A}$, we find that using $\nu=0$. a uniaxial negative strain in the horizontal direction (uniaxial 


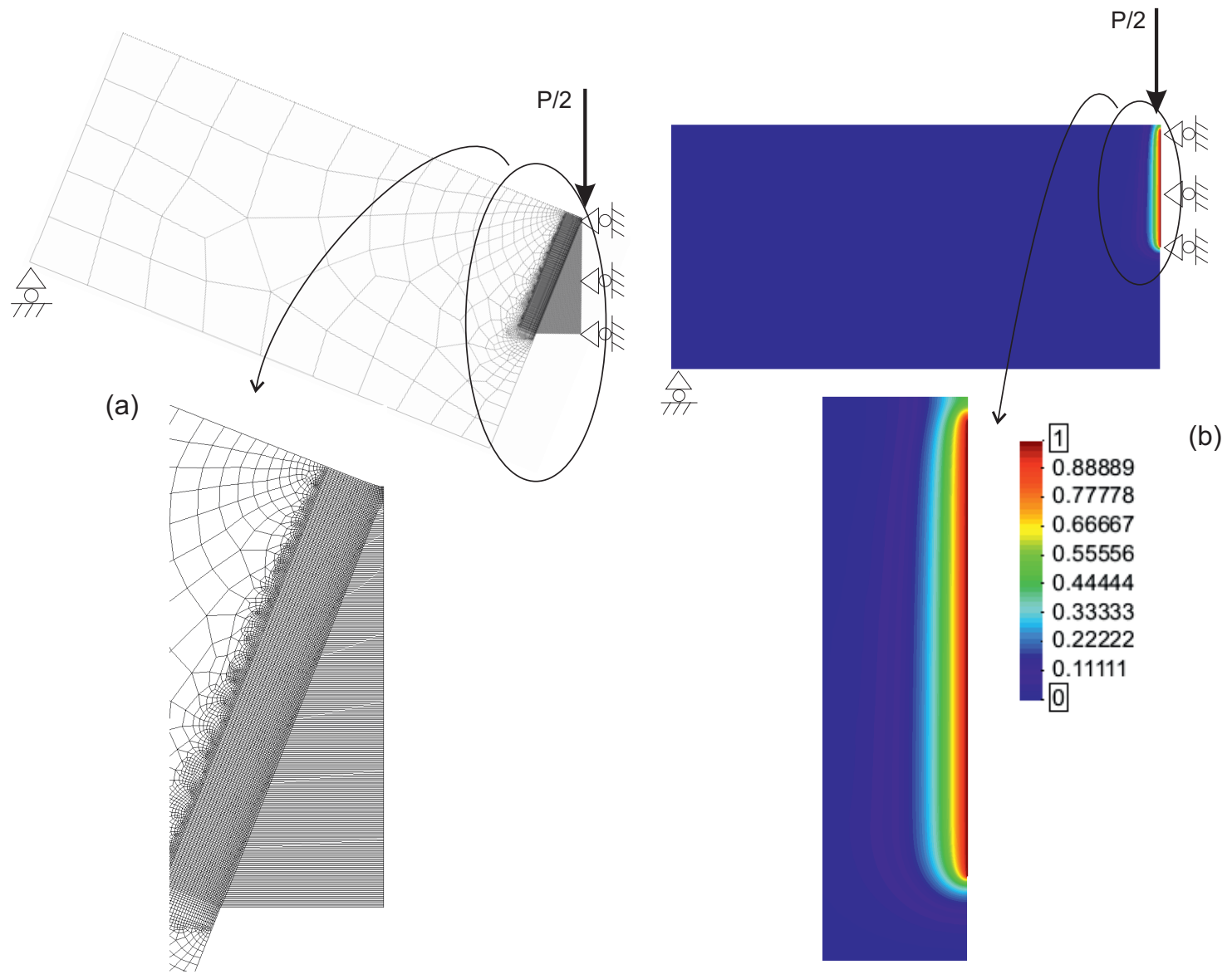

Figure 9: SENB test. Results at the end of analysis. (a) Deformed specimen. (b) Damage distribution. 
compressive stress) is obtained. Alternatively, with $\nu=0.25$, a biaxial strain is computed in that zone, being the larger principal strain in the horizontal direction negative, but a small positive strain (with vertical component $\left.\left(\mathbf{E}_{e}\right)_{y y}\right)$ is also computed. The positive component $\left(\mathbf{E}_{e}\right)_{y y}$ is the responsible for producing an increase (even small) of $\varphi$. With this analysis, we remark that the strain triaxiality effects are included in the evolution of the damage model. This issue is relevant because it plays an important role when crack propagation problems are evaluated in ductile metals.

Table 4 shows the overestimation, in percent, of the total expended work to achieve the complete structural failure. In this case, the theoretical expended work is $62.5[\mathrm{~N} / \mathrm{mm}]$.

\begin{tabular}{|c|c|c|c|c|}
\hline & $l=0.9[\mathrm{~mm}]$ & $l=1.2[\mathrm{~mm}]$ & $l=1.8[\mathrm{~mm}]$ & $l=2.4[\mathrm{~mm}]$ \\
\hline $\begin{array}{c}\text { Mesh A } \\
h^{\mathfrak{e}}=0.05[\mathrm{~mm}]\end{array}$ & $8.6 \%$ & $7.8 \%$ & $7.2 \%$ & $6.8 \%$ \\
\hline $\begin{array}{c}\text { Mesh 1 } \\
h^{\mathfrak{e}}=0.1[\mathrm{~mm}]\end{array}$ & $11.8 \%$ & $9.5 \%$ & $7.8 \%$ & $7.3 \%$ \\
\hline $\begin{array}{c}\text { Mesh 2 } \\
h^{\mathfrak{e}}=0.3[\mathrm{~mm}]\end{array}$ & - & $25.1 \%$ & $17.6 \%$ & $14.3 \%$ \\
\hline \hline
\end{tabular}

Table 4: SENB test. Percentage of overestimation of the simulated external work to produce complete structural failure respect to the exact value.

After evaluating the total dissipated energy in both tests, DENT and SENB, the following conclusions can be pointed out:

i) The overestimation of the dissipated energy is notably high, even when fine meshes and controlled loading increment techniques are used. Thus, bilinear quadrilateral finite elements show a very slow convergence rate with mesh refinement;

ii) From the authors' experience, acceptable results can be obtained with meshes having finite element sizes $h^{\mathfrak{e}}$ smaller than $0.2 l$, even better if $h^{\mathfrak{e}} \leq 0.1 l$. From this conclusion, the result corresponding to Mesh $2, l=1.2[\mathrm{~mm}]$ could be considered as not reliable;

iii) It has not been possible to evaluate, with acceptable confidence, the dissipated energy sensitivity with respect to the parameter $l$.

\subsection{Mode I crack growth analysis under small-scale yielding conditions}

The numerical model developed previously to study the growth of a preexisting crack in an elasticplastic body under plane strain mode I loading is here applied. Our purpose is twofold:

- To investigate the effect of the material parameters, namely the elastic-plastic parameters $E$, $\nu, \sigma_{y}$ and $n$, and the phase-field parameters $g_{f}$ and $l$, on the fracture toughness.

- To study the nature of crack extension.

Towards this end, a circular region of the body containing the crack (a modified boundary layer) is considered, as illustrated in Figure 11-a. The boundary conditions are inferred from the solution of linear fracture mechanics. To guarantee the validity of this boundary condition, which involves 


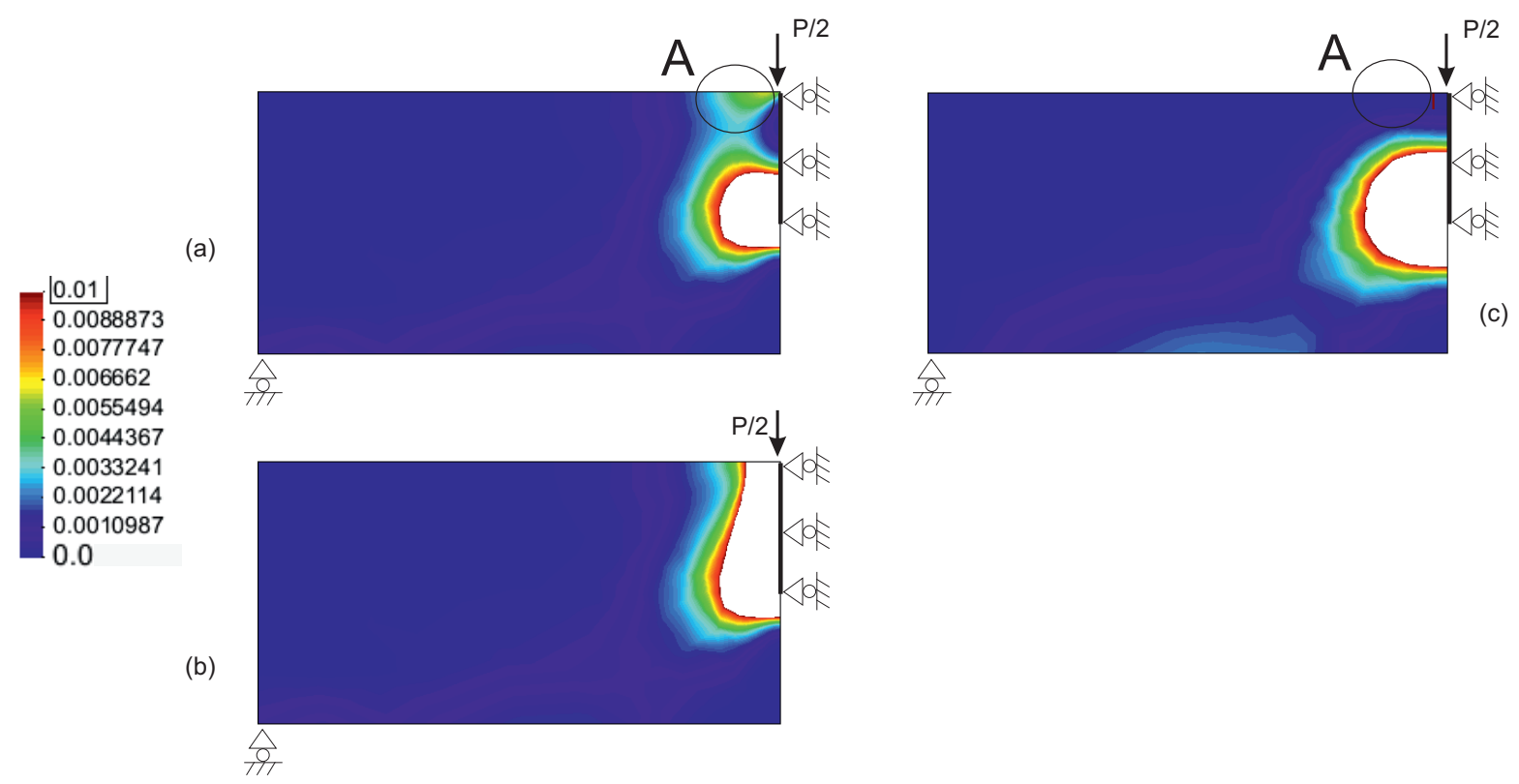

Figure 10: SENB test and sensitivity of strain triaxiality. Damage distribution. Values with $\varphi>0.01$ have been removed from the pictures. (a) At the initial stage, before reaching the structural limit point $(\nu=0.25)$. (b) At the end of analysis $(\nu=0.25)$. (c) At the initial stage, before reaching the structural limit point $(\nu=0$.).

the stress intensity factor $K_{I}$, the plastic and process zones developed around the crack tip remain small relative to the size of the circular region.

As the outcome of careful numerical simulations, the present model predicts, inter alia, that:

- For given $E, \nu, \sigma_{y}, n$ and $g_{f}$, fracture toughness, here denoted $K_{S S}$ and being the maximum $K_{I}$-value attained up to initiation of crack growth, increases as $l$ decreases. In particular, the simulation indicates that there is a critical value of $l$, say $l_{c}$, such that $K_{S S} \rightarrow \infty$ as $l \rightarrow l_{c}$. Thus, if $l \leq l_{c}$ the theory predicts that crack expansion is impossible no matter the load level. Therefore, to describe crack expansion, a finite value of $l$ greater than $l_{c}$ must be taken. This value of $l$ can be estimated provided the experimental value for $K_{S S}$ is known.

- In some cases, the advance of the pre-existent crack involves the following steps: 1) formation of a new crack ahead the original crack tip; 2) propagation of the new crack towards, and also away, the tip of the original crack; 3) extension of original crack when its tip is reached by the new crack. This implies the crack extension can occur in a discontinuous manner. This mechanism has been invoked for explaining brittle fracture in elastic-plastic solids (c.f. Tetelman and McEvily [31]; Kfouri [32]; Kfouri and Miller [33]; Ritchie et al. [23]). Interestingly, this effect is captured even using a small strain deformation theory.

\subsubsection{Mechanical model}

The geometrical description of the test and finite element model are displayed in Figure 11. Due to the problem symmetry, only one half (the upper part) of the domain is modeled. A structured mesh of BBAR finite elements of size $\left(h^{\mathfrak{e}} \times h^{\mathfrak{e}}\right)$ are used in the vicinity of the crack tip, see Figure 11-b. The structured mesh extends over a domain of size: $90 h^{\mathfrak{e}} \times 40 h^{\mathfrak{e}}$ around the crack tip. The 
finite element size is: $h^{\mathfrak{e}}=40 .[\mu \mathrm{m}]$. Note that the complete geometry of the problem, including the notch size, is parametrized with $h^{\mathfrak{e}}$.

Displacements, defined by the K-field around a sharp crack loaded in mode I, are prescribed on the far boundary $A-B-C$. They are given by:

$$
\begin{aligned}
& u_{x}=\frac{2(1+\nu) K_{I}}{E} \sqrt{\frac{r}{2 \pi}} \cos (\theta / 2)\left[1-2 \nu+\sin ^{2}(\theta / 2)\right] \\
& u_{y}=\frac{2(1+\nu) K_{I}}{E} \sqrt{\frac{r}{2 \pi}} \sin (\theta / 2)\left[2-2 \nu-\cos ^{2}(\theta / 2)\right]
\end{aligned}
$$

with $(r, \theta)$ being the polar coordinates of the boundary points and $K_{I}$ is the stress intensity factor. The coordinate system is centered in the crack tip. The displacements of boundary $C-D$ are prescribed to satisfy symmetry conditions.

The initial value and boundary conditions imposed on the phase field are: $\varphi=0$ on the complete domain, $\nabla \varphi \cdot \mathbf{n}=0$ on the boundary $D-A-B-C$.

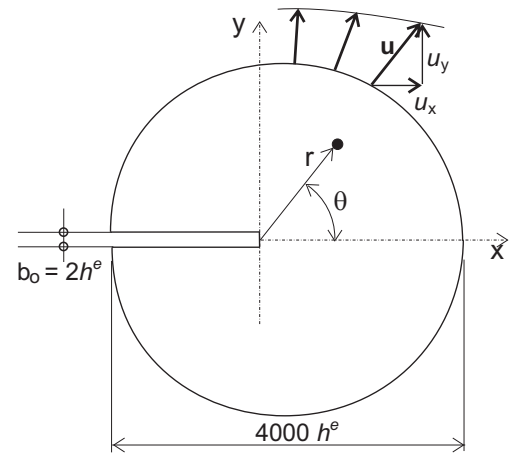

(a)

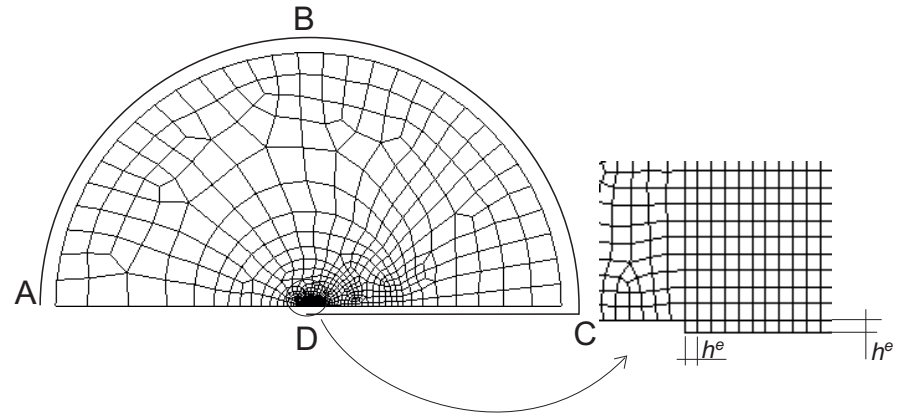

(b)

Figure 11: Mode I crack growth. (a) Geometry data. (b) Finite element model.

In the following simulations, the far boundary conditions are prescribed by controlling the crack mouth opening displacement through the parameter $\Delta s$, as shown in Figure 13-a.

Material parameters: the yield stress is taken: $\sigma_{y}=10[\mathrm{MPa}]$, the ratio between Young's modulus and yield stress is $E / \sigma_{y}=333.3$, Poisson's ratio: $\nu=0.3$. The strain hardening exponent $n$ changes according to the analyzed cases. The material parameters of the phase-field model are defined in the following.

\subsubsection{Plasticity and phase-field models: analysis of the interaction effects}

According to Tvergaard and Hutchinson [47], the two most important adimensional parameters governing the interaction effects between the mechanisms of crack separation and plastic deformation, are:

i) the ratio $\hat{\sigma} / \sigma_{y}$, where $\hat{\sigma}$ is the critical traction of the crack separation mechanism, i.e. the maximum traction of the traction-separation law describing the cohesive forces acting through the crack surfaces; and $\sigma_{y}$ is the yield stress of the plastic model; 
ii) the strain hardening exponent $n$ of the plastic model.

The phase-field model has not an explicit parameter identifying the critical traction $\hat{\sigma}$. However, it can be estimated through the parameters $g_{f}$ and $l$ as follows. First, a uniaxial (one-dimensional) homogeneous elastic phase-field problem is analytically solved. The equations governing this problem are the momentum balance equations (TI-1), the phase-field equation (TI-2) and the constitutive equation (TI-3) (with $\mathbf{E}_{e}=\mathbf{E}$ ) described in Table 1. Due to the assumed homogeneity, all gradients are zero $(\nabla \varphi=\Delta \varphi=0)$. Then, the result of this problem, reported in Ciarbonetti et al. [48] and Borden et al. [49], provides stress-strain responses like those plotted in Figure 12. They are plotted for several fracture energies and length parameters. Finally, the maximum stress values of those responses, and the corresponding strains, are given by the following equations:

$$
\hat{\sigma}=\frac{3 \sqrt{3}}{16} \sqrt{\frac{g_{f} E}{l}} \quad ; \quad \hat{\varepsilon}=\frac{1}{\sqrt{3}} \sqrt{\frac{g_{f}}{l E}}
$$

Note that, due to the assumed spatial homogeneity of the 1-D problem, the response given by equation (91) is highly idealized and artificial because strain localization is precluded, and thus, it does not represent any possible physical solution of a crack problem. Therefore, the area enclosed by the curves in Figure 12, a density of energy per unit of volume uniformly distributed in the 1D domain, is not related with the parameter $g_{f}$.

In any case, expression (91)-a allows to estimate $\hat{\sigma}$ as a suitable, but crude, assessment of the separation critical stress value provided by the phase-field model. Note the similarity between (91) and the classical expression of the Irwin's charactersitic lenght given in (92).

\section{Characterization of interaction effects through the parameter $g_{f}$}

Similar to the analysis reported in Tvergaard and Hutchinson [47] using a cohesive model, the crack growth resistance provided by the joint interacting models of plasticity and phase-field is next analyzed. This study takes the parameters $g_{f}$ and $n$ as two variables, while holding fixed $l, E$ and $\sigma_{y}$. Instead of considering $l \rightarrow 0$, as would be required for recovering the sharp-crack limit theory in elastic solids, we fix $l$ with a not null value, being: $l=400[\mu \mathrm{m}]=10 h^{e}$. The reason of adopting this decision are given in the present analysis, where we show that $l$ must be reinterpreted as a material parameter.

The fracture energy $g_{f}$ defines the critical stresses $\hat{\sigma}\left(g_{f}\right)$ through equation (91). Once $\hat{\sigma}\left(g_{f}\right)$ and $n$ are given, the analysis of the fracture toughness of the propagating crack is evaluated. In the following analysis, we have varied the parameter $g_{f}$.

Note that, considering the plastic parameters for this case, the classical estimation of the reference plastic zone size (Irwin's characteristic length) is:

$$
R_{0}=\frac{1}{3 \pi\left(1-\nu^{2}\right)} \frac{g_{f} E}{\sigma_{y}^{2}}
$$

Considering fracture energies ranging from $g_{f}=[0.116,1.024][\mathrm{N} / \mathrm{mm}]$, the corresponding range of $\hat{\sigma}\left(g_{f}\right) / \sigma_{y}$ and $R_{0}$ are: $\hat{\sigma}\left(g_{f}\right) / \sigma_{y}=[1,3]$ and $R_{0}=[450,3970].[\mu m]$. Then, from these figures, the notch tip of the numerical model has an initial width, ranging from $2 h^{e}=0.18 R_{0}$ (for the ratio $\hat{\sigma} / \sigma_{y}=1$ ) to $2 h^{e}=0.02 R_{0}$ (for the ratio $\hat{\sigma} / \sigma_{y}=3$ ). We can conclude that, for high fracture energies, the numerical model satisfies the sharp crack assumption. 

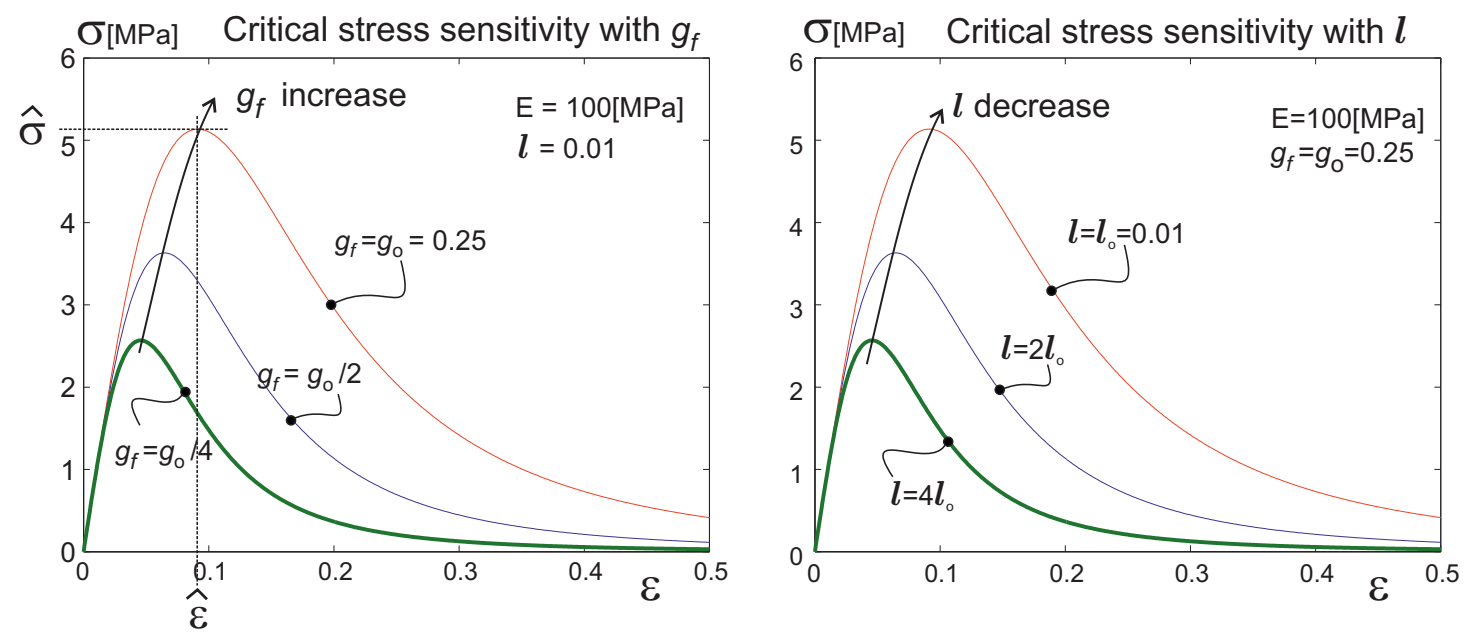

Figure 12: Phase-field model critical stress value as a function of $g_{f}[\mathrm{~N} / \mathrm{mm}]$ and $l[\mathrm{~mm}]$, for a homogeneous onedimensional problem.

Finally, notice that the ratio $\hat{\sigma} / \sigma_{y}$, governing the interaction effects between the phase-field model and plasticity, is proportional to $\sqrt{R_{0} / l}$. So, the following discussion given in terms of the former could be rephrased in terms of the later.

\section{Results obtained with the numerical model}

Figure 13 displays results obtained with the parameters $n=0$ (perfect plasticity) and $g_{f}=$ $0.328[\mathrm{~N} / \mathrm{mm}]\left(\hat{\sigma}\left(g_{f}\right) / \sigma_{y}=1.7\right)$.

Figure 13-b plots the vertical displacement $u_{y}^{T}$ of point T (see Figure 13-a) vs. control parameter $\Delta s$ (i.e. the crack mouth opening displacement) normalized with $h^{\mathfrak{e}}$. In the inserts of the same Figure (Figure 13-c:f) are displayed the evolution of damage $\varphi$, with $\varphi>0.97$. Arbitrarily, we consider that points with $\varphi>0.97$ represents the crack. Every picture is in correspondence with a point identified in the equilibrium curve: $u_{y}^{T}$ vs. $\Delta s / h^{e}$.

Figure 13-g plots the vertical tensile stress $\left(S_{y y}\right)$ normalized with the yield stress along the horizontal symmetry $\mathrm{x}$-axis. A sequence of plots are depicted while the maximum damage value remains below to 0.97 . The plot denoted $\mathrm{D}$ coincides with the instant of crack initiation. Note that the position of the crack initiation point does not coincide with the maximum tensile stress point along the x-axis. But, it is located in the elastic/plastic interface, as shown in Figure 13-c. Also with low damage values (plot A), note that the point with maximum tensile stress is located in the notch tip. But, with higher loads (plots B and C), the point with maximum tensile stress changes toward the interior of the body. Similar responses have been reported in classical elasto plastic solutions for crack propagation (c.f. McMeecking [50], Ritchie et al. [23]). Those studies necessarily took into account geometry changes of the root notch. Here, we assume that the finite width notch root geometry has not changed in the analysis.

After the crack initiates, the crack expands in both directions along the horizontal axis. Also, it is noted that the crack initiates when the vertical displacement $u_{y}^{T}$ has decreased from the maximum value $\left(\left(u_{y}^{T}\right)_{\max }\right)$ obtained in previous loading steps. Not in all cases the crack initiates in the interior of the body. In a few cases, the crack starts to propagate from the notch root. 

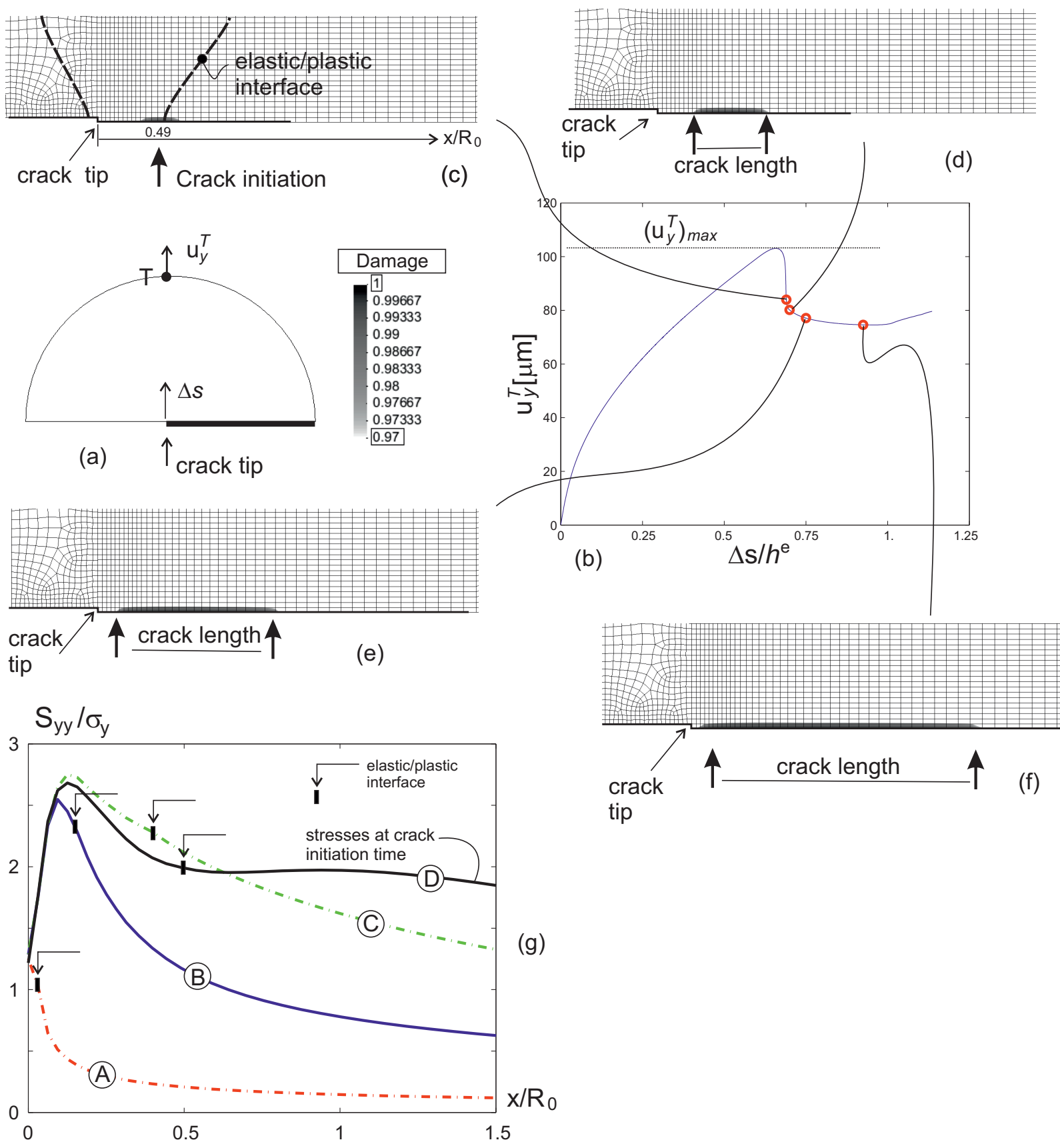
tip

Figure 13: Crack evolution. Case: $n=0, \hat{\sigma} / \sigma_{y}=1.7, R_{0}=1270$. $[\mu \mathrm{m}]$. (a) Control load parameter $\Delta s$ and Point $\mathrm{T}$ where the vertical displacement $u_{y}^{T}$ is evaluated as a function of $s$. (b) Plot of $u_{y}^{T}$ vs $\Delta s$. (c-f) Sequence of pictures displaying the damage distribution during the loading process (only values with $\varphi>0.97$ are shown). (g) Distribution of vertical tensile stresses $\left(S_{y y}\right)$, normalized with the yield stress, along the horizontal symmetry axis at different stages: $\mathrm{D}$ is at the time of crack initiation as shown in figure (c), and $\mathrm{A}, \mathrm{B}$ and $\mathrm{C}$ are in previous times when maximum damage values are $0.003,0.06$ and 0.18 , respectively. 
The stress intensity factor $K_{I}$ is determined from $\left(u_{y}^{T}\right)$ and equation (90). Figure 14 displays two curves of $K_{I}$ versus the crack mouth opening $\Delta s$. They are defined for $n=0$., $\hat{\sigma} / \sigma_{y}=0.5$ and $n=0.2, \hat{\sigma} / \sigma_{y}=3$, respectively. In those plots, the stress intensity factor $K_{I}$ is normalized with the fracture toughness in the absence of plasticity: $K_{0}=\left(E g_{f}\right) /\left(1-\nu^{2}\right)$. The horizontal axis corresponds to the crack mouth opening displacement, $\Delta s$, normalized with $h^{\mathfrak{e}}$. We recall that the steady-state material toughness $K_{S S}$ is defined as: $K_{S S}=K_{I}\left(\left(u_{y}^{T}\right)_{\max }\right)$. In other words, $K_{S S}$ is the maximum value of $K_{I}$ attained during crack propagation.
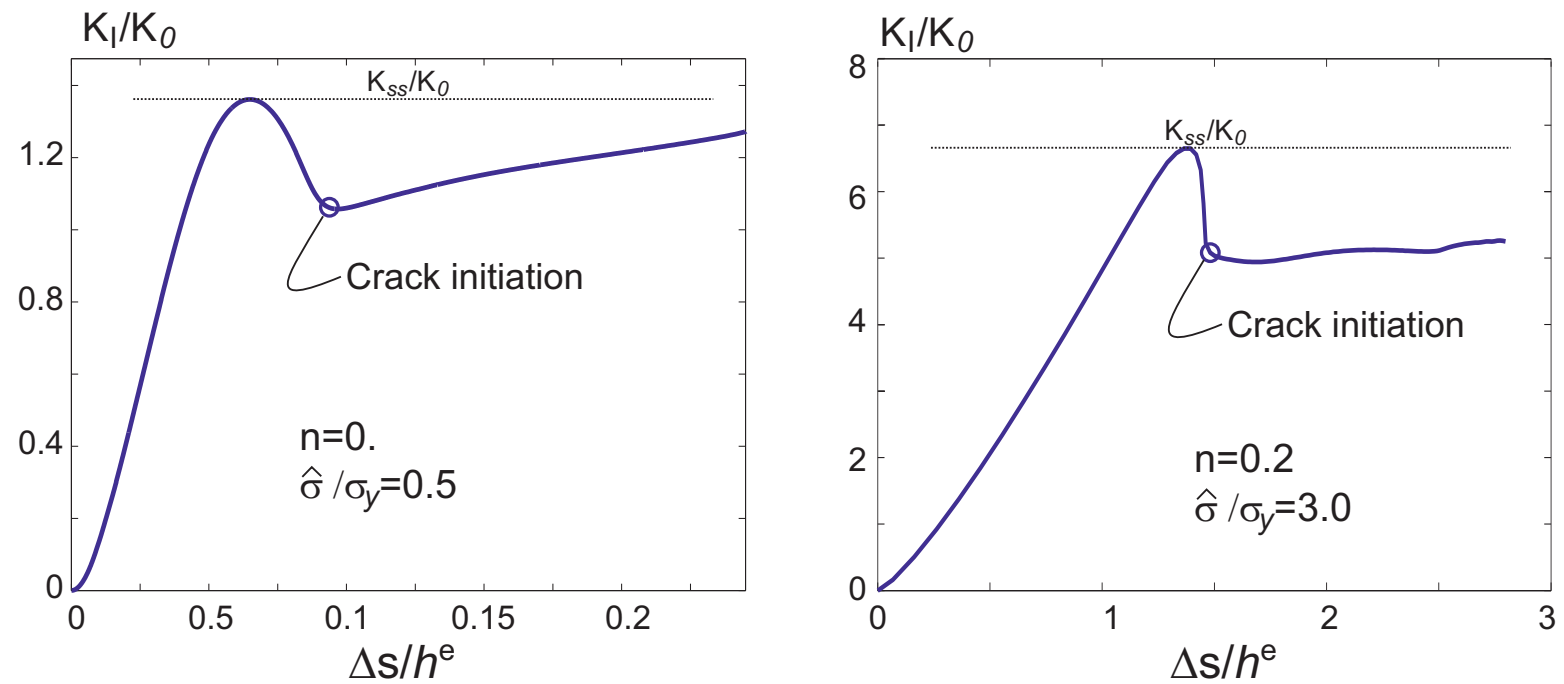

Figure 14: Mode I crack growth. Normalized $K_{I}$ vs. crack opening displacements. a) $n=0.0, \hat{\sigma} / \sigma_{y}=0.5$. b) $n=0.2, \hat{\sigma} / \sigma_{y}=3.0$.

Figure 15 displays the set of results obtained with different values of the ratio $\hat{\sigma}\left(g_{f}\right) / \sigma_{y}$ and taking $n=0$ and $n=0.2$, respectively. The vertical axis corresponds to the steady-state toughness $K_{S S}$ normalized with $K_{0}$. The abscissa represents the ratio $\hat{\sigma}\left(g_{f}\right) / \sigma_{y}$. Comparing the responses displayed by both curves with the results presented in the paper of Tvergaard and Hutchinson, we conclude that the phase field model interacts with the plastic model in a similar way as the cohesive model does, under the condition that $l$ stay fixed.

\subsubsection{Discussion of results}

The results obtained with perfect plasticity $(n=0)$ and $\hat{\sigma} / \sigma_{y}>2$ do not display crack propagation even considering the effect introduced by the phase-field model. A vertical asymptote for these responses is observed close to $\hat{\sigma} / \sigma_{y}=2$. In all cases satisfying $\hat{\sigma} / \sigma_{y}>2$, the maximum calculated value for damage, in the complete problem domain, remains low $(\varphi<0.2)$. The classical blunted notch response without crack surface separation is captured. In the work of Tvergaard and Hutchinson, a similar asymptotic value is obtained for $\hat{\sigma} / \sigma_{y}=3$, which agrees with theoretical predictions. The difference for capturing the asymptotic value between both models lies on the fact that $\hat{\sigma}$, in the phase-field model, represents a crude estimation of the separation critical stress. A similar tendency to capture a blunted notch without crack formation is observed when the strain hardening exponent is nonzero $(n=0.2)$ and $g_{f} \rightarrow \infty$.

Alternatively, the elastic sharp crack limit response is reached when plasticity is not developed. This happens if the phase-field model limits the stresses to be lower than $\sigma_{y}$. According 


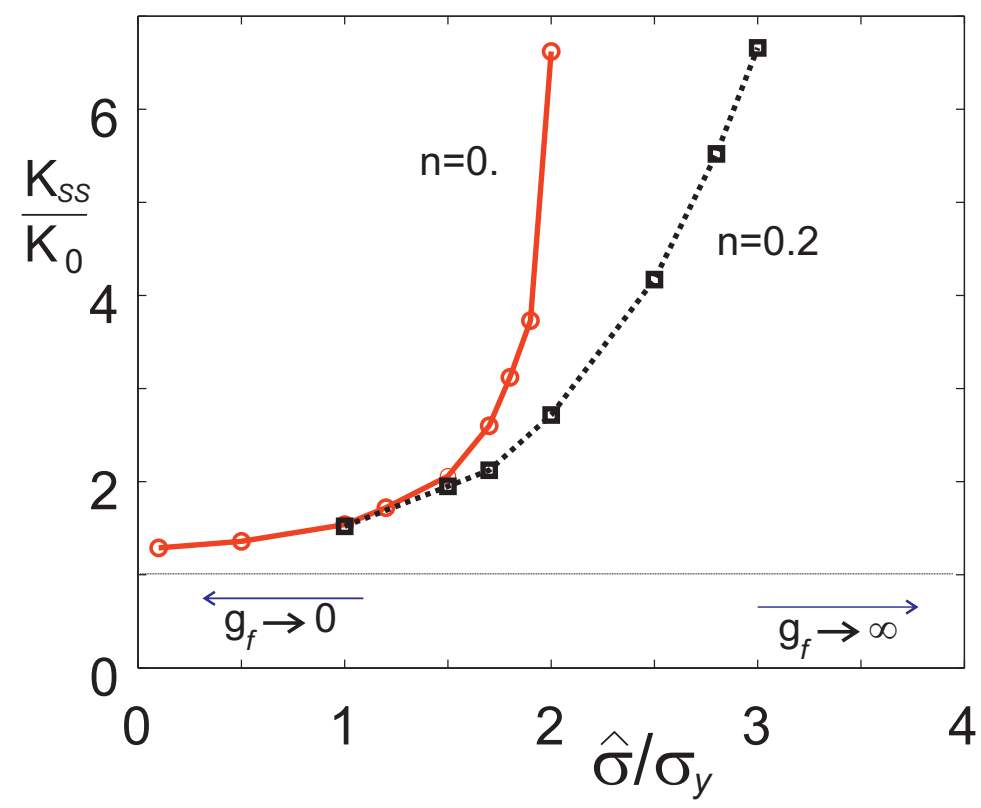

Figure 15: Mode I crack growth. $K_{S S}$ stress intensity factor as a function of $\hat{\sigma} / \sigma_{y}$.

with equation (91), the maximum stress of the phase-field model can be estimated through $\hat{\sigma}$. Thus, if the parameter $l$ is fixed and nonzero, the sharp-crack elastic limit solution is achieved when $g_{f} \rightarrow 0$. As observed in Figure 15, this limit is reached when: $\hat{\sigma} / \sigma_{y} \rightarrow 0$. Note that the limit value $K_{S S} / K_{0}=1$ is only approximately captured by the model. From the tests presented in sub-Sections 4.1.2 and 4.1.3, we know that very refined finite element meshes are needed to approximate accurately the dissipated energy in the sharp-crack elastic limit solution (and thus, reaching the limit $K_{S S} / K_{0}=1$ ).

Consider now the case $l \rightarrow 0$ while holding fixed the remaining parameters. As it is known, by taking a purely elastic response without plasticity, the model asymptotically tends to the sharp crack solution of the linear elastic fracture mechanics theory. However, when the phase-field is coupled with a plastic model, with $l \rightarrow 0$ and $g_{f}$ staying fixed, $\hat{\sigma} \rightarrow \infty$, as shown by equation (91). Thus, the criterion for crack activation is never achieved. And the blunted notch solution is unavoidably captured. This happens even taking $n>0$.

From this observation, it is concluded that the length $l$ has to be taken as a material non-null parameter which, together with $g_{f}$ and the plastic model parameters, must be properly characterized. The fact of considering two parameters defining the phase-field model is equivalent to what happens with cohesive approaches, where two parameters must be empirically defined, such as the critical stress separation and fracture energy.

\section{Conclusions}

In the first part of this paper, we formulated a phase-field theory of fracture in elastic-plastic solids under small strains, where a scalar damage variable is introduced and treated as an independent kinematical descriptor. The governing equations of the theory are obtained by combining the force balances, introduced via the principle of virtual power, and a thermodynamically consistent consti- 
tutive theory that allows for general coupling between elasticity, classical plasticity and damage. A special version of the theory suitable to the description of rate-independent plasticity and damage is presented. The phase-field model combined with plasticity is suitable to analyze the role played by plastic deformations during the surface separation phenomena observed in crack propagation problems.

In the second part of this work, the finite element formulation is presented and several numerical assessments are performed. From the studied tests, some conclusions can be drawn, which are listed in the following items:

i) When phase-field models are coupled with plasticity, the length scale $l$ has to be reinterpreted. Thus, aside from the plastic parameters, the phase-field model has to be characterized through two parameters: the fracture energy $g_{f}$ and the length scale parameter $l$. Alternatively, and equivalently to cohesive models, instead of taking $l$, the critical stress $\hat{\sigma}$ of the crack separation phenomena can be adopted as the parameter to be characterized, while $l$ is estimated through equation (91)-a.

ii) Similar to cohesive approaches, the present phase-field model is able to capture solutions displaying strain localization bands leading to strong discontinuity kinematics. This property has been highlighted in the numerical solutions presented in Section 4. The reader is referred to Appendix II for additional comments related to this topic.

iii) Different from cohesive approaches, the phase-field model takes into account the strain triaxiallity to determine the stresses across the surface where the crack is processing. As can be seen from the phase-field equation (12), the evolution of the phase field variable depends on the elastic energy, which in turn, depends on the strain triaxility. Instead, cohesive models lack of this dependence. This conclusion is an extremly important issue when ductile fracture problems are simulated.

Finally, from a numerical point of view, an important issue related to finite element technologies has to be remarked. The crack path determined with a phase-field model is automatically detected, once the variable $\varphi$ has been evaluated. The path can arbitrarily intersect the finite element mesh. A major deficiency is related to the computational cost, because the model provides accurate solution only by taking very fine meshes. The finite elements have to be small enough, as small as the order of the parameter $l$.

\section{Acknowledgments}

The first author gratefully acknowledges financial support provided by CAPES (PCPP 004/2011) and CNPq (312153/2013-9).

The research leading to these results has received funding from the European Research Council under the European Unions Seventh Framework Programme (FP/2007-2013) / ERC Grant Agreement n. 320815 (ERC Advanced Grant Project Advanced tools for computational design of engineering materials COMP-DES-MAT).

The Joint Research Project Mercosur (CAPES, Brazil, SPU, Argentina) has also provided additional financial support. 


\section{Appendix I: Sketch to demonstrate the equivalence between phase-field equa- tions (56) and (69)}

We have not a formal proof of the equivalence between equations (56) and (69). However, under mild assumptions, we can sketch partial proofs showing that solution of equation (69) satisfies some basic requirements placed by the phase-field equations. Furthermore, numerical experiments have shown results coherent with the statements below.

\section{- $\underline{\text { Statement } 1}$}

Consider a body $\mathcal{B}$ undergoing a damage process driven by $h:=\hat{\psi}_{e}^{+}\left(\mathbf{E}_{\mathrm{e}}\right)$. Then, under mild assumption, the phase-field equation satisfies:

a) $\varphi(t)=\varphi\left(t_{0}\right)$ for all $t \in\left(t_{0}, t_{1}\right)$ provided that $h(s) \leq h\left(t_{0}\right)$ for all $s \in\left(t_{0}, t\right)$.

b) $h(t)>h(s)$ for all $s \in\left(t_{0}, t\right)$ implies that $\dot{\varphi}(t) \neq 0$.

- $\underline{\text { Statement } 2}$

(valid for an infinite domain with $\varphi=0$ for $\mathbf{x} \rightarrow \infty$ and assuming $l \mathcal{H} / g_{f} \ll 1$ )

Given equation (69) and: $\dot{\mathcal{H}}(\mathbf{x})>0 \quad \Rightarrow \quad \dot{\varphi} \geq 0$ in all $\mathcal{B}$.

\section{Proof of Statement 1}

From (56) and (TI-4), it follows that $\varphi$ and $\pi_{\mathrm{r}}$ obey

$$
\pi_{\mathrm{r}} \leq 0, \quad \pi_{\mathrm{r}}=2(1-\varphi) h-\frac{g_{f}}{l}\left(\varphi-l^{2} \triangle \varphi\right) .
$$

We intend to discuss some features of the solutions of (AI-1) for a given driving force history $h$. Before doing that, we make the following remark:

- Suppose that $\left(\left(\pi_{\mathrm{r}}\right)_{1}, \varphi_{1}, h_{1}\right)$ and $\left(\left(\pi_{\mathrm{r}}\right)_{2}, \varphi_{2}, h_{2}\right)$ satisfy (AI-1). Then, a single calculation shows that the condition $\varphi_{1}=\varphi_{2}$ in a vicinity of an arbitrary point of $\mathcal{B}$ implies that

$$
h_{2} \leq h_{1}-\frac{\left(\pi_{\mathrm{r}}\right)_{1}}{2\left(1-\varphi_{1}\right)}
$$

in that vicinity. Equivalently, if the condition

$$
h_{2}>h_{1}-\frac{\left(\pi_{\mathrm{r}}\right)_{1}}{2\left(1-\varphi_{1}\right)}
$$

holds in a vicinity of an arbitrary point of $\mathcal{B}$, then $\varphi_{1} \neq \varphi_{2}$ in that vicinity. 
We now return to discussion related to the evolution of $\varphi$ in an vicinity of an arbitrary point of $\mathcal{B}$ during a process starting at $t=t_{0}$ and ending at $t=t_{1}$. Suppose that $\varphi, \pi_{r}$ and $h$ are known at $t_{0}$, which are respectively given by $\varphi\left(t_{0}\right), \pi_{r}\left(t_{0}\right) \equiv 0$ and $h\left(t_{0}\right)$, and that $h$ is prescribed in the interval $\left(t_{0}, t_{1}\right)$. All the quantities are assumed to be smooth. Under these conditions, Statement 1 follows from remarks leading to (AI-2) and (AI-3). Statement 1, by its turn, suggests that (69) holds in the interval $\left(t_{0}, t_{1}\right)$.

\section{Proof of Statement 2}

Consider an infinite domain in $\mathbf{R}^{3}$ which is denoted $\mathcal{B}$, and define the problem:

$$
\begin{array}{rr}
F^{H}=2\left(1-\varphi^{H}\right) \mathcal{H}(\mathbf{x}, t)-\frac{g_{f}}{l}\left(\varphi^{H}-l^{2} \triangle \varphi^{H}\right)=0 ; & \text { in } \quad \mathcal{B} \\
\mathcal{H}(\mathbf{x}, t)=\max _{s} \hat{\psi}_{e}^{+}(\mathbf{x}, s), & s \in[0, t] \\
\varphi^{H}=0 \quad \text { for } & \mathbf{x} \rightarrow \infty ;
\end{array}
$$

where $\mathcal{H}(\mathbf{x}, t)$ satisfies: $\dot{\mathcal{H}}(\mathbf{x}, t) \geq 0$. After assuming that the parameter $l$ is very small, and such that:

$$
\frac{l \mathcal{H}}{g_{f}} \ll 1
$$

equation $(\mathrm{AI}-4)$ can be rewritten in rates as follows:

$$
\begin{array}{r}
\Delta \dot{\hat{\varphi}}-\frac{1}{l^{2}} \dot{\hat{\varphi}}=-\chi(\mathbf{x}, t) ; \\
\chi(\mathbf{x}, t)=2 \frac{(1-\hat{\varphi})}{l g_{f}} \dot{\mathcal{H}} \geq 0 \\
\dot{\hat{\varphi}}=0 \quad \text { for } \quad \mathbf{x} \rightarrow \infty ;
\end{array}
$$

Equation (AI-6) is a linear differential equation (Screened Poisson Equation) which solution can be found through the integral:

$$
\dot{\hat{\varphi}}(\mathbf{x}, t)=\int_{\mathcal{B}} G\left(\mathbf{x}-\mathbf{x}_{0}\right) \chi\left(\mathbf{x}_{0}, t\right) d v
$$

where $G\left(\mathbf{x}-\mathbf{x}_{0}\right)$ is the Green's function of the problem (AI-6) with the right hand side term being replaced by: $\chi(\mathbf{x}, t)=\delta\left(\mathbf{x}_{0}\right)\left(\delta\left(\mathbf{x}_{0}\right)\right.$ is the Dirac's delta function). In $\mathbf{R}^{3}$ :

$$
G(\mathbf{x})=\frac{1}{4 \pi x} e^{-x / l^{2}} ; \quad x=|\mathbf{x}| ;
$$

Since: $G \geq 0$ and $\chi\left(\mathbf{x}_{0}, t\right) \geq 0$ in all $\mathcal{B}$ and $t \in\left[0, t_{f}\right]$, we conclude that:

$$
\dot{\hat{\varphi}} \geq 0 ; \quad \text { in } \quad \mathcal{B} ; t \in\left[0, t_{f}\right]
$$




\section{Appendix II: Comparative analysis for modeling strong discontinuities with phase-field versus a specific class of gradient damage model}

In this Appendix we elaborate a brief discussion excluding plasticity effects, comparing the present phase-field model with the so-called implicit damage-gradient model reported by Peerlings et al. [46]. This implicit damage-gradient model can be considered as a specific class of gradient damage models.

The point here discussed raises important implications for the numerical modeling of fracture, and is specifically addressed to compare the intrinsic capability for modeling Traction free Strong Discontinuities within a continuum setting. Rigorous analysis is not pursued. But, the attention is emphasized on a physical interpretation of the additional terms in the Screened Poisson-type equation describing the damage distribution, as well as in the authors' numerical experience using them.

Similar to the phase-field model for fracture adopted in this contribution, the implicit damagegradient model of Peerlings et al. postulates that the mechanical problem can be stated in terms of two independent variables $\left\{\boldsymbol{u}, \tilde{\varepsilon}_{e q}\right\}$, where $\boldsymbol{u}$ is the displacement vector field and $\tilde{\varepsilon}_{e q}$ is an equivalent "non-local" strain measure. The variable $\tilde{\varepsilon}_{e q}$ is a function of the "local" equivalent strain counterpart $\varepsilon_{e q}(\boldsymbol{u}):=1 / 2\left(\lambda\langle\operatorname{tr} \mathbf{E}\rangle^{2}+2 \mu \mathbf{E}: \mathbf{E}\right)$, defined through the following Screened Poisson-type differential equation (which is written in rates):

$$
\frac{\hat{l}^{2}}{2} \Delta \dot{\tilde{\varepsilon}}_{e q}-\dot{\tilde{\varepsilon}}_{e q}=-\overbrace{\dot{\varepsilon}_{e q}(\boldsymbol{u})}^{\dot{F}_{d r i v i n g}^{d g} \geq 0} \text { in } \mathcal{B} \quad ; \quad \nabla \dot{\tilde{\varepsilon}}_{e q} \cdot \boldsymbol{n}=0 \quad \text { in } \partial \mathcal{B} ;
$$

where $\hat{l}$ is a length scale parameter. In this gradient-based formulation the damage variable depends on $\tilde{\varepsilon}_{e q}$.

For comparison purposes, the Screened Poisson-type equation for the phase-field model is additionally re-written (in rates) as follows:

$$
l^{2} \Delta \dot{\varphi}-\dot{\varphi}=-\left(\frac{2 l}{g_{f}} \overline{(1-\varphi) \mathcal{H}(\boldsymbol{u})}\right) \quad \text { in } \mathcal{B} \quad ; \quad \nabla \dot{\varphi} \cdot \boldsymbol{n}=0 \quad \text { in } \partial \mathcal{B}
$$

and therefore:

$$
l^{2} \Delta \dot{\varphi}-\left(1+\frac{2 l \mathcal{H}(\boldsymbol{u})}{g_{f}}\right) \dot{\varphi}=-\overbrace{\frac{2 l}{g_{f}}(1-\varphi) \overline{\mathcal{H}(\boldsymbol{u})}}^{\dot{F}_{\text {driving }}^{\text {pf }} \geq 0} \quad \text { in } \mathcal{B} \quad ; \quad \nabla \dot{\varphi} \cdot \boldsymbol{n}=0 \quad \text { in } \partial \mathcal{B} ;
$$

The right-hand sides of (AII-1) and (AII-3), denoted as $\dot{F}_{d r i v i n g}^{d g}$ and $\dot{F}_{d r i v i n g}^{p f}$, represent the rate driving forces for the implicit-damage-gradient and the phase-field models, respectively. Note that both approaches share similar mathematical structure incorporating a length scale parameter, $\hat{l}$ and $l$, respectively.

However, some important differences can be marked. Strong discontinuity kinematics is characterized by a singular strain on the crack, here denoted $\mathcal{S}$. Due to this singular kinematics, both terms, $\varepsilon_{e q}(\boldsymbol{u})$ and $\mathcal{H}(\boldsymbol{u})$ in the right-hand side of (AII-1) and (AII-3), tend to infinity on $\mathcal{S}$. Notice 
however that since $\varphi$ grows to 1 on $\mathcal{S}$, the total rate driving force of the phase-field model, $\dot{F}_{d r i v i n g}^{p f}$, goes to zero as a direct consequence of the quadratic form, in the term $(1-\varphi)$, assumed in the definition of the elastic free energy density $\hat{\psi}_{e}$, see equation (59). On the crack, the variable $\varphi$ reaches the maximum value $(\varphi=1)$ and stop to grow, and $\dot{F}_{d r i v i n g}^{p f}=0$. Thus a real traction free boundary is captured using the phase-field model in total correspondence with the strong discontinuity model.

Alternatively, the rate driving force of the gradient-based formulation, $\dot{F}_{d r i v i n g}^{d g}$, remains almost singular, and therefore the non-local strain $\tilde{\varepsilon}_{e q}$ grows unbounded. The damage variable increase until reaching the maximum value, 1 , however due to the characteristic evolution of $\dot{F}_{d r i v i n g}^{d g}$, damage can be spuriously spread from the crack to the surrounding medium. The spurious transference of damage introduces numerical difficulties for capturing a strong discontinuity kinematics. A finite zone around the crack, involving several finite elements in the discrete model, can reach total degradation. Thus, strong discontinuity regimes can not be properly modeled in the implicit continuum damage-gradient environment. Solutions to this issue have been proposed in the literature (c.f. Comi et al. [51]).

Using the present phase-field model, the authors have not detected this kind of numerical difficulties. Particularly, see the results in Figure 6.

\section{References}

[1] J.R. Rice. A path independent integral and the approximate analysis of strain concentration by notches and cracks. Technical report, DTIC Document, 1967.

[2] M. E Gurtin and P. Podio-Guidugli. Configurational forces and the basic laws for crack propagation. J. Mech. Phys. Solids, 44(6):905-927, 1996.

[3] J. Tillberg, F. Larsson, and K. Runesson. On the role of material dissipation for the crackdriving force. Int. J. Plast., 26(7):992-1012, 2010.

[4] J.R. Rice. Mechanics of quasi-static crack growth. Technical report, Brown Univ., Providence, RI (USA). Div. of Engineering, 1978.

[5] N.K. Simha, F.D. Fischer, G.X. Shan, C.R. Chen, and O. Kolednik. j--integral and crack driving force in elastic-plastic materials. J. Mech. Phys. Solids, 56(9):2876-2895, 2008.

[6] W. Brocks, A. Cornec, and I. Scheider. Computational aspects of nonlinear fracture mechanics. GKSS Forshungszentrum Geesthacht GMBH-Publications-GKSS, (30), 2003.

[7] H. Stumpf, J. Makowski, and K. Hackl. Dynamical evolution of fracture process region in ductile materials. Int. J. Plast., 25(5):995-1010, 2009.

[8] J.C. Simo, J. Oliver, and F. Armero. An analysis of strong discontinuities induced by strainsoftening in rate-independent inelastic solids. Comp. Mech., 12(5):277-296, 1993.

[9] T. Belytschko and R. Gracie. On xfem applications to dislocations and interfaces. Int. J. Plast., 23(10):1721-1738, 2007. 
[10] J. Oliver, A.E. Huespe, M.D.G. Pulido, and E. Chaves. From continuum mechanics to fracture mechanics: the strong discontinuity approach. Eng. Fract. Mech., 69(2):113-136, 2002.

[11] A.E Huespe, A. Needleman, J Oliver, and P.J. Sánchez. A finite thickness band method for ductile fracture analysis. Int. J. Plast., 25(12):2349-2365, 2009.

[12] A.E. Huespe, A. Needleman, J. Oliver, and P.J. Sánchez. A finite strain, finite band method for modeling ductile fracture. Int. J. Plast., 28(1):53-69, 2012.

[13] G. Pijaudier-Cabot and Z.P. Bazant. Nonlocal damage theory. Journal of Engineering Mechanics, 113(10):1512-1533, 1987.

[14] M. Frémond and B. Nedjar. Damage, gradient of damage and principle of virtual power. Int. J. Solids Struc., 33(8):1083-1103, 1996.

[15] J. Lemaitre. Local approach of fracture. Eng. Fract. Mech., 25(5):523-537, 1986.

[16] I.S. Aranson, V.A. Kalatsky, and V.M. Vinokur. Continuum field description of crack propagation. Phys. Rev. Letters, 85(1):118, 2000.

[17] R. Spatschek, E. Brener, and A. Karma. Phase field modeling of crack propagation. Phil. Magazine, 91:7595, 2011.

[18] M.N. Silva Jr., F.P. Duda, and E. Fried. Sharp-crack limit of a phase-field model for brittle fracture. J. Mech. Phys. Solids, 61(11):2179-2195, 2013.

[19] V. Hakim and Al. Karma. Laws of crack motion and phase-field models of fracture. J. Mech. Phys. Solids, 57(2):342-368, 2009.

[20] G.A. Francfort and J.J. Marigo. Revisiting brittle fracture as an energy minimization problem. J. Mech. Phys. Solids, 46(8):1319-1342, 1998.

[21] A. Karma and A.E. Lobkovsky. Unsteady crack motion and branching in a phase-field model of brittle fracture. J. Mech. Phys. Solids, 92(24):245510, 2004.

[22] H. Henry and H. Levine. Dynamic instabilities of fracture under biaxial strain using a phase field model. Phys. Rev. Letters, 93(10):105504, 2004.

[23] R.O. Ritchie, J.F. Knott, and J.R. Rice. On the relationship between critical tensile stress and fracture toughness in mild steel. J. Mech. Phys. Solids, 21(6):395-410, 1973.

[24] G. Capriz. Continua with Microstructure. Springer, 1989.

[25] E. Fried and M.E. Gurtin. Continuum theory of thermally induced phase transitions based on an order parameter. Phys D, 68:326-343, 2003.

[26] B. Bourdin, G.A. Francfort, and J.J. Marigo. Numerical experiments in revisited brittle fracture. J. Mech. Phys. Solids, 48(4):797-826, 2000. 
[27] C. Miehe, M. Hofacker, and F. Welschinger. A phase field model for rate-independent crack propagation: Robust algorithmic implementation based on operator splits. Comput. Meth. App. Mech. Eng., 199(45):2765-2778, 2010.

[28] B Nedjar. Elastoplastic-damage modelling including the gradient of damage: formulation and computational aspects. International Journal of Solids and Structures, 38(30):54215451, 2001.

[29] O. Aslan, N.M. Cordero, A. Gaubert, and S. Forest. Micromorphic approach to single crystal plasticity and damage. Int. J. of Eng. Sci., 49(12):1311-1325, 2011.

[30] K. Saanouni and M. Hamed. Micromorphic approach for finite gradient-elastoplasticity fully coupled with ductile damage: Formulation and computational aspects. Int. J. Solids Struc., 50(14):2289-2309, 2013.

[31] A.S. Tetelman and A.J. McEvily. Fracture of structural materials, volume 264. Wiley New York, 1967.

[32] A.P. Kfouri. Characteristic crack-tip distances in fracture criteria: Is crack propagation discontinuous? Eng. Fract. Mech., 75(7):1815-1828, 2008.

[33] A.P. Kfouri and K.J. Miller. Crack separation energy rates in elastic-plastic fracture mechanics. Proc. of the Inst. of Mech. Eng., 190(1):571-584, 1976.

[34] V.I. Levitas. Structural changes without stable intermediate state in inelastic material. part ii. applications to displacive and diffusional-displacive phase transformations, strain-induced chemical reactions and ductile fracture. Int. J. Plast., 16(7):851-892, 2000.

[35] A.V. Idesman, V.I. Levitas, and E. Stein. Structural changes in elastoplastic material: a unified finite-element approach to phase transformation, twinning and fracture. Int. J. Plast., 16(7): 893-949, 2000.

[36] M.E. Gurtin, E. Fried, and L. Anand. The mechanics and thermodynamics of continua. Cambridge University Press, 2010.

[37] B.D. Coleman and W. Noll. The thermodynamics of elastic materials with heat conduction and viscosity. Arch. Ration. Mech. Anal., 13:167-178, 1963.

[38] J.C. Simo and T.J. Hughes. Computational Inelasticity. New York, NY: Springer New York, 1998.

[39] E.C. Aifantis. The physics of plastic deformation. Int. J. Plast., 3(3):211-247, 1987.

[40] B. Bourdin, G.A. Francfort, and J.J. Marigo. The variational approach to fracture. Journal of Elasticity, 91(1-3):5-148, 2008.

[41] L. Ambrosio and V.M. Tortorelli. Approximation of functional depending on jumps by elliptic functional via t-convergence. Comm. Pure Appl. Math., 43(8):999-1036, 1990. 
[42] T. Liebe, P. Steinmann, and A. Benallal. Theoretical and computational aspects of a thermodynamically consistent framework for geometrically linear gradient damage. Comput. Meth. App. Mech. Eng., 190:6555-6576, 2001.

[43] C.V. Verhoosel and René de Borst. A phase-field model for cohesive fracture. Int. J. Num. Meth. Eng., 2013.

[44] A. Simone. Continuous-discontinuous modelling of failure. 2003. PhD Thesis, TU Delft, Delft University of Technology.

[45] C. Kuhn and R. Müller. A continuum phase field model for fracture. Eng. Fract. Mech., 77: 3625-3634, 2010.

[46] R. Peerlings, R. de Borst, W. Brekelmans, and J. de Vree. Gradient enhanced damage for quasi-brittle materials. Int. J. Num. Meth. Eng., 39:3391-3403, 1996.

[47] V. Tvergaard and J.W. Hutchinson. The relation between crack growth resistance and fracture process parameters in elasto-plastic solids. J. Mech. Phys. Solids, 40:1377-1397, 1992.

[48] A. Ciarbonetti, A.E. A.E. Huespe, P.J. Sánchez, and M.N da Silva. Modelo de campo de fase para simular fráctura frágil. Mecánica Computacional, Vol XXXI, pages 1655-1670, 2012.

[49] M.J. Borden, C. V. Verhoosel, M. A. Scott, T. J. R. Hughes, and C. M. Landis. A phasefield description of dynamic brittle fracture. ICES REPORT 11-14, 2011. The Institute for Computational Engineering and Sciences, The University of Texas at Austin.

[50] R.M. McMeecking. Finite deformation analysis of crack-tip opening in elastic-plastic materials and implications for fracture. J. Mech. Phys. Solids, 25:357-381, 1977.

[51] C. Comi, S. Mariani, and U. Perego. An extended fe strategy for transition from continuum damage to mode i cohesive crack propagation. Int. J. Num. Meth. Eng., 31(2):213-238, 2007. 